\title{
Higher-order relativistic corrections to gluon fragmentation into spin-triplet $S$-wave quarkonium
}

\author{
Geoffrey T. Bodwin, ${ }^{a}$ U-Rae $\mathrm{Kim}^{b}$ and Jungil $\mathrm{Lee}^{b}$ \\ ${ }^{a}$ HEP Division, Argonne National Laboratory, \\ 9700 South Cass Avenue, Argonne, IL 60439, U.S.A. \\ ${ }^{b}$ Department of Physics, Korea University, \\ Seoul 136-713, Korea. \\ E-mail: gtb@hep.anl.gov, sadafada@korea.ac.kr, jungil@korea.ac.kr
}

\begin{abstract}
We compute the relative-order- $v^{4}$ contribution to gluon fragmentation into quarkonium in the ${ }^{3} S_{1}$ color-singlet channel, using the nonrelativistic QCD (NRQCD) factorization approach. The QCD fragmentation process contains infrared divergences that produce single and double poles in $\epsilon$ in $4-2 \epsilon$ dimensions. We devise subtractions that isolate the pole contributions, which ultimately are absorbed into long-distance NRQCD matrix elements in the NRQCD matching procedure. The matching procedure involves two-loop renormalizations of the NRQCD operators. The subtractions are integrated over the phase space analytically in $4-2 \epsilon$ dimensions, and the remainder is integrated over the phase-space numerically. We find that the order- $v^{4}$ contribution is enhanced relative to the order- $v^{0}$ contribution. However, the order- $v^{4}$ contribution is not important numerically at the current level of precision of quarkonium-hadroproduction phenomenology. We also estimate the contribution to hadroproduction from gluon fragmentation into quarkonium in the ${ }^{3} P_{J}$ color-octet channel and find that it is significant in comparison to the complete next-to-leading-order-in- $\alpha_{s}$ contribution in that channel.
\end{abstract}

KEYWORDS: quarkonium, fragmentation, NRQCD, relativistic corrections

ARXIV EPRINT: 1208.5301 


\section{Contents}

1 Introduction 1

2 Collins-Soper definition of the fragmentation function 4

3 NRQCD factorization $\quad 6$

3.1 NRQCD factorization formulas for $g \rightarrow J / \psi$ through order $v^{4} \quad 8$

$\begin{array}{lll}4 & \text { Kinematics } & 9\end{array}$

5 Full-QCD calculations $\quad 10$

$\begin{array}{ll}5.1 \quad D_{0}\left[g \rightarrow Q \bar{Q}\left({ }^{3} S_{1}^{[8]}\right)\right] & 13\end{array}$

$\begin{array}{lll}5.2 & D_{2}\left[g \rightarrow Q \bar{Q}\left({ }^{3} P^{[8]}\right)\right] & 13\end{array}$

$\begin{array}{ll}5.3 \quad D_{4}\left[g \rightarrow Q \bar{Q}\left({ }^{3} S_{1}^{[1]}\right)\right] & 15\end{array}$

6 NRQCD LDMEs 18

$\begin{array}{lll}6.1 & \text { Order } \alpha_{s}^{0} & 18\end{array}$

$\begin{array}{lll}6.2 \text { Order } \alpha_{s} & 18\end{array}$

$\begin{array}{ll}6.2 .1{ }^{3} P^{[8]} \rightarrow{ }^{3} S_{1}^{[1]} & 18\end{array}$

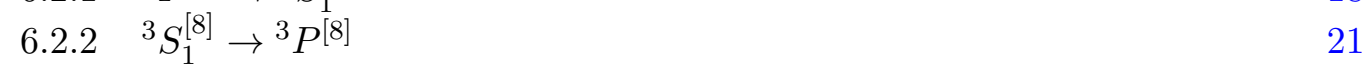

$\begin{array}{lll}6.3 & \text { Order } \alpha_{s}^{2} & 21\end{array}$

6.4 Renormalization-group evolution 24

$\begin{array}{lll}7 & \text { NRQCD matching and short-distance coefficients } & 25\end{array}$

$\begin{array}{lll}8 & \text { Numerical results } & 27\end{array}$

$\begin{array}{lll}9 & \text { Summary } & 31\end{array}$

$\begin{array}{ll}\text { A Analytic calculation of phase-space integrals } & 33\end{array}$

B Parametrizations of the short-distance coefficients 36

\section{Introduction}

Heavy-quarkonium production in hard-scattering collisions has a long and rich history of experimental measurements and theoretical calculations [1]. Intense efforts in this area are expected to continue as the Large Hadron Collider (LHC) makes available data with unprecedented momentum transfers and statistics.

In recent years, a great deal of theoretical effort has been focused on the nonrelativistic QCD (NRQCD) factorization approach [2] to calculations of quarkonium production rates. 
In this approach, it is conjectured that the inclusive quarkonium production cross section at large transverse momentum $\left(p_{T}\right)$ can be written as a sum of products of short-distance coefficients and long-distance matrix elements (LDMEs):

$$
\sigma(H)=\sum_{n} F_{n}\left(\mu_{\Lambda}\right)\left\langle 0\left|\mathcal{O}_{n}^{H}\left(\mu_{\Lambda}\right)\right| 0\right\rangle .
$$

Here, $\mu_{\Lambda}$ is the factorization scale, which is the cutoff of the effective field theory NRQCD. A short-distance coefficient $F_{n}\left(\mu_{\Lambda}\right)$ is, in essence, the partonic cross section to produce a heavy-quark-antiquark $(Q \bar{Q})$ pair with certain quantum numbers, convolved with parton distribution functions. The short-distance coefficients can be calculated as perturbation series in the strong-coupling constant $\alpha_{s}$. A production LDME $\left\langle 0\left|\mathcal{O}_{n}^{H}\left(\mu_{\Lambda}\right)\right| 0\right\rangle$ is the probability for a $Q \bar{Q}$ pair with certain quantum numbers to evolve into a particular heavy-quarkonium state. It is expressed as the vacuum expectation value of a four-fermion operator

$$
\mathcal{O}_{n}^{H}\left(\mu_{\Lambda}\right)=\left\langle 0\left|\chi^{\dagger} \kappa_{n} \psi \mathcal{P}_{H(P)} \psi^{\dagger} \kappa_{n}^{\prime} \chi\right| 0\right\rangle,
$$

where $\psi^{\dagger}$ and $\chi$ are two-component (Pauli) fields that create a heavy quark and a heavy antiquark, respectively, and $\kappa_{n}$ and $\kappa_{n}^{\prime}$ are combinations of Pauli and color matrices. ${ }^{1}$

$$
\mathcal{P}_{H(P)}=\sum_{X}|H(P)+X\rangle\langle H(P)+X|
$$

is a projection onto a state consisting of a quarkonium $H$, with four-momentum $P$, plus anything. $\mathcal{P}_{H(P)}$ contains a sum over any quarkonium polarization quantum numbers that are not specified explicitly. The NRQCD LDMEs are evaluated in the rest frame of the quarkonium, in which $P=(M, \mathbf{0})$, where $M$ is the quarkonium mass. In the remainder of this paper, we suppress the momentum argument of $\mathcal{P}_{H(P)}$ in NRQCD LDMEs.

The production LDMEs for the evolution of color-singlet $Q \bar{Q}$ pairs into a quarkonium state are related to the color-singlet quarkonium decay LDMEs. These color-singlet production LDMEs can be determined from comparison of theory with quarkonium production or decay data or from lattice QCD calculations. However, the production LDMEs for the evolution of color-octet $Q \bar{Q}$ pairs into a quarkonium state can be determined, at least at present, only through comparison of theory with experimental quarkonium-production data.

Complete calculations of short-distance coefficients in the NRQCD factorization approach now exist through next-to-leading order (NLO) in $\alpha_{s}$ for production of the $J / \psi$ and the $\psi(2 S)$ in $e^{+} e^{-}$collisions, in $e p$ collisions, and in $p \bar{p}$ and $p p$ collisions $[1,5-15]$. These calculations include the contributions from all of the color-octet channels through relative order $v^{4}$, as well as the contribution of the color-singlet channel at leading order (LO) in $v$. Specifically, the calculations include the contributions of the ${ }^{3} S_{1},{ }^{1} S_{0}$ and ${ }^{3} P_{J}$ color-octet channels and the ${ }^{3} S_{1}$ color-singlet channel at the leading nontrivial order in $v$ in each channel. Here, $v$ is half the relative velocity of the heavy quark and the heavy

\footnotetext{
${ }^{1}$ It was pointed out by Nayak, Qiu, and Sterman that gauge invariance requires that the definitions of the NRQCD LDMEs include Wilson lines that run from the quark and antiquark fields to infinity [3, 4]. For simplicity, we have omitted these Wilson lines here.
} 
antiquark in the quarkonium rest frame. $v^{2} \approx 0.22$ for the $J / \psi$, and $v^{2} \approx 0.1$ for the $\Upsilon$. These theoretical results are generally compatible with experimental measurements of quarkonium production cross sections. However, significant discrepancies remain between theoretical predictions for quarkonium polarization and experimental measurements [1315]. These discrepancies might point to as-yet-uncalculated theoretical contributions, to experimental difficulties, to a failure of convergence of the NRQCD series in $\alpha_{s}$ or $v$, or to a failure of the NRQCD factorization conjecture itself.

The calculations at NLO in $\alpha_{s}$ have revealed very large corrections in that order to the ${ }^{3} P_{J}$ color-octet channel and the ${ }^{3} S_{1}$ color-singlet channel. Large corrections have also been found in a calculation of the real-emission contributions to $\Upsilon$ hadroproduction at next-to-next-to-leading order in $\alpha_{s}$ [16]. The large corrections are the result of kinematic enhancements of the higher-order cross sections at high $p_{T}$ relative to the LO cross sections. The sizes of these corrections have cast some doubt on the convergence of the perturbation series.

It has been suggested recently that the large higher-order corrections to quarkonium production can be brought under control by re-organizing the perturbation series according to the $p_{T}$ behavior of the various contributions [17]. In this approach, the cross section can be shown to factorize into convolutions of hard-scattering cross sections with fragmentation functions. The factorization holds up to corrections of relative order $m_{c}^{4} / p_{T}^{4}$, where $m_{c}$ is the charm-quark mass. The factorized cross section consists of a leading contribution, which arises from single-particle fragmentation into a quarkonium and falls as $1 / p_{T}^{4}$ in the partonic cross section and a first subleading contribution, which arises from two-particle fragmentation into a quarkonium and falls as $1 / p_{T}^{6}$ in the partonic cross section. If NRQCD factorization holds, then the various fragmentation functions can be expressed in terms of a sum of products of short-distance coefficients and NRQCD LDMEs. This picture has been shown to account for the large corrections at NLO in $\alpha_{s}$ in the ${ }^{3} S_{1}$ color-singlet channel [18].

In this paper, we compute the NRQCD short-distance coefficient for gluon fragmentation into a ${ }^{3} S_{1}$ color-singlet $Q \bar{Q}$ pair in relative order $v^{4}$. The short-distance coefficients for gluon fragmentation in this channel have been computed in relative order $v^{0}[19,20]$ and relative order $v^{2}[21]$. In both cases, the contributions are not important phenomenologically. Nevertheless, it is worthwhile to consider the order- $v^{4}$ contribution for two reasons. First, this contribution is interesting theoretically because it is in order $v^{4}$ that the ${ }^{3} S_{1}$ color-singlet fragmentation channel first develops soft divergences in full QCD. As we shall see, these soft divergences in full QCD correspond to soft divergences in the LDMEs for the ${ }^{3} S_{1}$ and ${ }^{3} P_{J}$ color-octet channels and cancel in the short-distance coefficients, as is required by NRQCD factorization. A second motivation for examining the order- $v^{4}$ contribution is that it is potentially large. Contributions from gluon fragmentation into the ${ }^{3} S_{1}$ and ${ }^{3} P_{J}$ color-octet channels are known to be important phenomenologically. The ${ }^{3} S_{1}$ color-singlet channel mixes with these channels in order $v^{4}$, and the partitioning of the various contributions is controlled by single and double logarithms of the factorization scale. Therefore, it is plausible that the order- $v^{4}$ contributions to the color-singlet channel could be large.

Our method of calculation is based on the Collins-Soper definition [22] of the frag- 
mentation function for a gluon fragmenting into a quarkonium. We assume that NRQCD factorization holds, that is, that the fragmentation function can be decomposed into a sum of products of short-distance coefficients and NRQCD LDMEs. We then compute the full-QCD fragmentation functions for a gluon fragmenting into free $Q \bar{Q}$ states with various quantum numbers. The ultimate aim is to match these full-QCD fragmentation functions to the corresponding NRQCD fragmentation functions in order to determine the NRQCD short-distance coefficients. Some additional details of this approach can be found in Ref. [21].

This paper is organized as follows. We give the Collins-Soper definition of the fragmentation function in Sec. 2. Section 3 contains the NRQCD factorization formula for the fragmentation function and also contains a discussion of the NRQCD LDMEs and short-distance coefficients that are relevant through relative order $v^{4}$. The kinematics and variables that we use in our calculation are described in Sec. 4. In Sec. 5, we discuss the calculation of the fragmentation processes in full QCD. As we have mentioned, an important feature of the present calculation is that soft divergences arise in the ${ }^{3} S_{1}$ color-singlet channel in both full QCD and NRQCD. These divergences ultimately cancel in the short-distance coefficients when we carry out the matching between full QCD and NRQCD. In both full QCD and NRQCD, we regulate the divergences dimensionally. In the case of full $\mathrm{QCD}$, we devise subtractions that remove the divergent terms from the integrand, and we compute the subtraction contributions analytically. This computation is described in Sec. 5.3. After we remove the subtraction terms, we calculate the remainder of the full-QCD contribution in four dimensions, carrying out the integration numerically. We compute the relevant NRQCD LDMEs for free $Q \bar{Q}$ states analytically in dimensional regularization. These calculations are described in Sec. 6. We also determine the evolution equations for the LDMEs and find a discrepancy with a result in Ref. [23]. In Sec. 7, we match the NRQCD and full-QCD fragmentation functions to obtain the short-distance coefficients, and we present numerical results for them in Sec. 8. Finally, in Sec. 9, we summarize our results.

\section{Collins-Soper definition of the fragmentation function}

Here, and throughout this paper, we use the following light-cone coordinates for a fourvector $V$ :

$$
\begin{aligned}
V & =\left(V^{+}, V^{-}, \boldsymbol{V}_{\perp}\right)=\left(V^{+}, V^{-}, V^{1}, V^{2}\right), \\
V^{+} & =\left(V^{0}+V^{3}\right) / \sqrt{2}, \\
V^{-} & =\left(V^{0}-V^{3}\right) / \sqrt{2} .
\end{aligned}
$$

The scalar product of two four-vectors $V$ and $W$ is then

$$
V \cdot W=V^{+} W^{-}+V^{-} W^{+}-\boldsymbol{V}_{\perp} \cdot \boldsymbol{W}_{\perp} .
$$


The Collins-Soper definition for the fragmentation function for a gluon fragmenting into a hadron (quarkonium) $H[22]$ is

$$
\begin{aligned}
D[g \rightarrow H]\left(z, \mu_{\Lambda}\right)= & \frac{-g_{\mu \nu} z^{d-3}}{2 \pi k^{+}\left(N_{c}^{2}-1\right)(d-2)} \int_{-\infty}^{+\infty} d x^{-} e^{-i k^{+} x^{-}} \\
& \times\left\langle 0\left|G_{c}^{+\mu}(0) \mathcal{E}^{\dagger}\left(0,0, \mathbf{0}_{\perp}\right)_{c b} \mathcal{P}_{H(P)} \mathcal{E}\left(0, x^{-}, \mathbf{0}_{\perp}\right)_{b a} G_{a}^{+\nu}\left(0, x^{-}, \mathbf{0}_{\perp}\right)\right| 0\right\rangle
\end{aligned}
$$

Here, $z$ is the fraction of the gluon's + component of momentum that is carried by the hadron, $G_{\mu \nu}$ is the gluon field-strength operator, $k$ is the momentum of the field-strength operator, $\mu_{\Lambda}$ is the factorization scale, and $d=4-2 \epsilon$ is the number of space-time dimensions. There is an implicit average over the color and polarization states of the initial gluon. The projection $\mathcal{P}_{H(P)}$ is given in Eq. (1.3). The fragmentation function is evaluated in the frame in which the hadron has zero transverse momentum: $P=\left[z k^{+}, M^{2} /\left(2 z k^{+}\right), \mathbf{0}_{\perp}\right]$. The operator $\mathcal{E}\left(0, x^{-}, \mathbf{0}_{\perp}\right)$ is a path-ordered exponential of the gluon field:

$$
\mathcal{E}\left(0, x^{-}, \mathbf{0}_{\perp}\right)_{b a}=\mathrm{P} \exp \left[+i g_{s} \int_{x^{-}}^{\infty} d z^{-} A^{+}\left(0, z^{-}, \mathbf{0}_{\perp}\right)\right]_{b a},
$$

where $g_{s}=\sqrt{4 \pi \alpha_{s}}$ is the QCD coupling constant and $A^{\mu}(x)$ is the gluon field. Both $A_{\mu}$ and $G_{\mu \nu}$ are $\mathrm{SU}(3)$ matrices in the adjoint representation. The expression (2.3) is manifestly gauge invariant. We use the Feynman gauge in our calculation.

The Feynman rules for the perturbative expansion of Eq. (2.3) are given in Ref. [22]. The quantity $\mathcal{E}\left(0, x^{-}, \mathbf{0}_{\perp}\right)$ appears in the Feynman rules as an eikonal line. Owing to the charge-conjugation properties of the $Q \bar{Q}$ states that we consider and the Landau-Yang theorem $[24,25]$, gluon attachments to the eikonal lines from $\mathcal{E}\left(0, x^{-}, \mathbf{0}_{\perp}\right)$ do not appear in our calculation. Hence, we need only the standard QCD Feynman rules, an overall factor

$$
C_{\text {frag }}=\frac{z^{d-3} k^{+}}{2 \pi\left(N_{c}^{2}-1\right)(d-2)}
$$

from Eq. (2.3), and the special Feynman rule for the vertex that creates a gluon and an eikonal line. That vertex is shown in Fig. 1. Its Feynman rule, in momentum space, is a factor

$$
+i\left(g^{\nu \alpha}-\frac{Q^{\nu} n^{\alpha}}{k^{+}}\right) \delta_{a b}
$$

where $k$ is the sum of the momenta of the gluon and the eikonal line, $Q$ is the momentum of the gluon, $\alpha$ is the polarization index of the gluon, and $a$ and $b$ are the color indices, respectively, of the gluon and the eikonal line. In the absence of interactions with the eikonal lines, $k=Q . n$ is a light-like vector whose components are given by $n=\left(0,1, \mathbf{0}_{\perp}\right)$.

The final-state phase space that is implied by Eq. (2.3) is

$$
d \Phi_{n}=\frac{4 \pi M}{S} \delta\left(k^{+}-P^{+}-\sum_{i=1}^{n} k_{i}^{+}\right) \theta\left(k^{+}\right) \prod_{i=1}^{n} \frac{d k_{i}^{+}}{4 \pi k_{i}^{+}} \frac{d^{d-2} \boldsymbol{k}_{i \perp}}{(2 \pi)^{d-2}} \theta\left(k_{i}^{+}\right)
$$




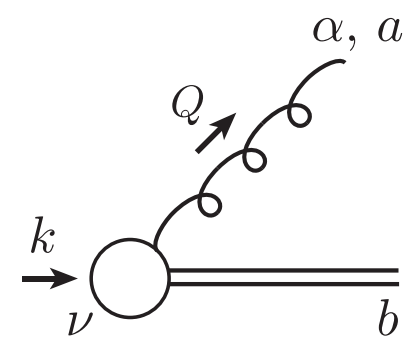

Figure 1. Feynman diagram for the vertex that creates a gluon and an eikonal line. The circle represents the operator $G_{a}^{+\nu}$, which creates a gluon with momentum $Q$ and polarization and color indices $\alpha$ and $a$, respectively. $b$ is the color index for the eikonal line. The operator momentum $k$ is the sum of $Q$ and the momentum of the eikonal line.

where $S$ is the statistical factor for identical particles in the final state, $k_{i}$ is the momentum of the $i$ th final-state particle, and the product is over all of the final-state particles except $H$. We use nonrelativistic normalization for the state $H$, and so a factor $2 M$ appears in the phase space in order to cancel the relativistic normalization of $H$ in the definition (2.3). We use relativistic normalization for all particles other than $H$.

\section{NRQCD factorization}

We assume that the fragmentation function for a gluon fragmenting into a quarkonium $H$ satisfies NRQCD factorization. Then, in analogy with Eq. (1.1), we have

$$
D[g \rightarrow H](z)=\sum_{n} d_{n}(z)\left\langle 0\left|\mathcal{O}_{n}^{H}\right| 0\right\rangle
$$

where the $\left\langle 0\left|\mathcal{O}_{n}^{H}\right| 0\right\rangle$ are NRQCD LDMEs and the $d_{n}(z)$ are the fragmentation short-distance coefficients. We have suppressed the dependences of $d_{n}(z)$ and $\left\langle 0\left|\mathcal{O}_{n}^{H}\right| 0\right\rangle$ on the factorization scale $\mu_{\Lambda}$. In discussing specific cases, we use the notation $\left.\langle 0| \mathcal{O}_{n_{v}, n^{\prime}}{ }^{2 s+1} l_{j}^{[c]}\right)|0\rangle$ for the LDMEs and the notation $d_{n_{v}, n^{\prime}}\left({ }^{2 s+1} l_{j}^{[c]}\right)(z)$ for the short-distance coefficients, where ${ }^{2 s+1} l_{j}$ is the standard spectroscopic notation for the angular-momentum quantum numbers of the corresponding NRQCD operator, and $c$ is the color quantum number of the NRQCD operator ( 1 or 8$). n_{v}$ is the order in $v$, relative to the leading order, of the field operators and derivatives in $\mathcal{O}_{n_{v}, n^{\prime}}^{H}$, excluding factors of $v$ from the projection onto the final state $H$. $n^{\prime}$ is an integer that is used to distinguish operators that have the same quantum numbers and order in $v$. We denote the contribution of order $v^{k}$ to $D[g \rightarrow H]$ by $D_{k}[g \rightarrow H]$.

We can write the fragmentation functions for gluon fragmentation into free $Q \bar{Q}$ states as

$$
D[g \rightarrow Q \bar{Q}](z)=\sum_{n} d_{n}(z)\left\langle 0\left|\mathcal{O}_{n}^{Q \bar{Q}}\right| 0\right\rangle .
$$

Since the short-distance coefficients $d_{n}(z)$ are independent of the specifics of the hadronic states, the $d_{n}(z)$ in Eq. (3.2) are identical to the $d_{n}(z)$ in Eq. (3.1). We determine the $d_{n}(z)$ by computing the left side of Eq. (3.2) in full QCD and comparing it with the right side, in which the free $Q \bar{Q}$ LDMEs are computed in NRQCD. Since we choose a factorization scale 
$\mu_{\Lambda}$ of order the heavy-quark mass $m$, we can carry out this computation in perturbation theory. We will denote the contribution of order $v^{k}$ to $D[g \rightarrow Q \bar{Q}]$ by $D_{k}[g \rightarrow Q \bar{Q}]$.

If $H$ is a ${ }^{3} S_{1}$ quarkonium state, such as the $J / \psi$, then, in LO in $v$, we must consider the LDME

$$
\left\langle 0\left|\mathcal{O}_{0}^{H}\left({ }^{3} S_{1}^{[1]}\right)\right| 0\right\rangle=\left\langle 0\left|\chi^{\dagger} \sigma^{i} \psi \mathcal{P}_{H} \psi^{\dagger} \sigma^{i} \chi\right| 0\right\rangle .
$$

In relative order $v^{2}$, we must consider the LDME

$$
\left\langle 0\left|\mathcal{O}_{2}^{H}\left({ }^{3} S_{1}^{[1]}\right)\right| 0\right\rangle=\frac{1}{2}\langle 0| \chi^{\dagger} \sigma^{i}\left(-\frac{i}{2} \stackrel{\leftrightarrow}{\boldsymbol{D}}\right)^{2} \psi \mathcal{P}_{H} \psi^{\dagger} \sigma^{i} \chi+\text { H. c. }|0\rangle .
$$

In relative order $v^{3}$, we must consider the LDME

$$
\left\langle 0\left|\mathcal{O}_{0}^{H}\left({ }^{1} S_{0}^{[8]}\right)\right| 0\right\rangle=\left\langle 0\left|\chi^{\dagger} T^{a} \psi \mathcal{P}_{H} \psi^{\dagger} T^{a} \chi\right| 0\right\rangle
$$

In relative order $v^{4}$, we must consider the LDMEs

$$
\begin{aligned}
& \left\langle 0\left|\mathcal{O}_{4,1}^{H}\left({ }^{3} S_{1}^{[1]}\right)\right| 0\right\rangle=\left\langle 0\left|\chi^{\dagger} \sigma^{i}\left(-\frac{i}{2} \stackrel{\leftrightarrow}{\boldsymbol{D}}\right)^{2} \psi \mathcal{P}_{H} \psi^{\dagger} \sigma^{i}\left(-\frac{i}{2} \stackrel{\leftrightarrow}{\boldsymbol{D}}\right)^{2} \chi\right| 0\right\rangle \\
& \left\langle 0\left|\mathcal{O}_{4,2}^{H}\left({ }^{3} S_{1}^{[1]}\right)\right| 0\right\rangle=\frac{1}{2}\langle 0| \chi^{\dagger} \sigma^{i}\left(-\frac{i}{2} \stackrel{\leftrightarrow}{\boldsymbol{D}}\right)^{4} \psi \mathcal{P}_{H} \psi^{\dagger} \sigma^{i} \chi+\text { H. c. }|0\rangle, \\
& \left\langle 0\left|\mathcal{O}_{4,3}^{H}\left({ }^{3} S_{1}^{[1]}\right)\right| 0\right\rangle=\frac{1}{2}\langle 0| \chi^{\dagger} \sigma^{i} \psi \mathcal{P}_{H} \psi^{\dagger} \sigma^{i}\left(\stackrel{\leftrightarrow}{D} \cdot g_{s} \boldsymbol{E}+g_{s} \boldsymbol{E} \cdot \stackrel{\leftrightarrow}{\boldsymbol{D}}\right) \chi \\
& -\chi^{\dagger} \sigma^{i}\left(\stackrel{\leftrightarrow}{\boldsymbol{D}} \cdot g_{s} \boldsymbol{E}+g_{s} \boldsymbol{E} \cdot \stackrel{\leftrightarrow}{\boldsymbol{D}}\right) \psi \mathcal{P}_{H} \psi^{\dagger} \sigma^{i} \chi|0\rangle, \\
& \left\langle 0\left|\mathcal{O}_{0}^{H}\left({ }^{3} S_{1}^{[8]}\right)\right| 0\right\rangle=\left\langle 0\left|\chi^{\dagger} \sigma^{i} T^{a} \psi \mathcal{P}_{H} \psi^{\dagger} \sigma^{i} T^{a} \chi\right| 0\right\rangle, \\
& \left\langle 0\left|\mathcal{O}_{0}^{H}\left({ }^{3} P_{0}^{[1]}\right)\right| 0\right\rangle=\frac{1}{d-1}\left\langle 0\left|\chi^{\dagger}\left(-\frac{i}{2} \stackrel{\leftrightarrow}{D} \cdot \boldsymbol{\sigma}\right) \psi \mathcal{P}_{H} \psi^{\dagger}\left(-\frac{i}{2} \stackrel{\leftrightarrow}{\boldsymbol{D}} \cdot \boldsymbol{\sigma}\right) \chi\right| 0\right\rangle, \\
& \left\langle 0\left|\mathcal{O}_{0}^{H}\left({ }^{3} P_{1}^{[1]}\right)\right| 0\right\rangle=\left\langle 0\left|\chi^{\dagger}\left(-\frac{i}{2} \stackrel{\leftrightarrow}{D^{[i} \sigma^{j]}}\right) \psi \mathcal{P}_{H} \psi^{\dagger}\left(-\frac{i}{2} \stackrel{\leftrightarrow}{D}^{[i} \sigma^{j]}\right) \chi\right| 0\right\rangle, \\
& \left\langle 0\left|\mathcal{O}_{0}^{H}\left({ }^{3} P_{2}^{[1]}\right)\right| 0\right\rangle=\left\langle 0\left|\chi^{\dagger}\left(-\frac{i}{2} \stackrel{\leftrightarrow}{D^{(i}} \sigma^{j)}\right) \psi \mathcal{P}_{H} \psi^{\dagger}\left(-\frac{i}{2} \stackrel{\leftrightarrow}{D^{(i}} \sigma^{j)}\right) \chi\right| 0\right\rangle
\end{aligned}
$$

Here, the symmetric traceless product is defined by

$$
A^{(i} B^{j)}=\frac{1}{2}\left(A^{i} B^{j}+A^{j} B^{i}\right)-\frac{1}{d-1} \delta^{i j} A^{k} B^{k},
$$

and the antisymmetric product is defined by

$$
A^{[i} B^{j]}=\frac{1}{2}\left(A^{i} B^{j}-A^{j} B^{i}\right) .
$$

For purposes of our calculation, it is also useful to define

$$
\begin{aligned}
\left\langle 0\left|\mathcal{O}_{0}^{H}\left({ }^{3} P^{[8]}\right)\right| 0\right\rangle & =\sum_{J=0,1,2}\left\langle 0\left|\mathcal{O}_{0}^{H}\left({ }^{3} P_{J}^{[8]}\right)\right| 0\right\rangle \\
& =\left\langle 0\left|\chi^{\dagger}\left(-\frac{i}{2} \stackrel{\leftrightarrow}{\boldsymbol{D}}\right)^{r} \sigma^{n} T^{a} \psi \mathcal{P}_{H} \psi^{\dagger}\left(-\frac{i}{2} \stackrel{\leftrightarrow}{\boldsymbol{D}}\right)^{r} \sigma^{n} T^{a} \chi\right| 0\right\rangle
\end{aligned}
$$

It was shown in Ref. [26] that, by making use of the NRQCD equations of motion, one can express the LDME $\left\langle 0\left|\mathcal{O}_{4,3}^{H}\left({ }^{3} S_{1}^{[1]}\right)\right| 0\right\rangle$ in terms of the LDMEs $\left\langle 0\left|\mathcal{O}_{4,1}^{H}\left({ }^{3} S_{1}^{[1]}\right)\right| 0\right\rangle$ and 
$\left\langle 0\left|\mathcal{O}_{4,2}^{H}\left({ }^{3} S_{1}^{[1]}\right)\right| 0\right\rangle$. (Equivalently, one can eliminate the LDME $\left\langle 0\left|\mathcal{O}_{4,3}^{H}\left({ }^{3} S_{1}^{[1]}\right)\right| 0\right\rangle$ by making use of a field redefinition [27].) Hence, we need not consider $\left\langle 0\left|\mathcal{O}_{4,3}^{H}\left({ }^{3} S_{1}^{[1]}\right)\right| 0\right\rangle$ in our analysis.

In the LDMEs $\left\langle 0\left|\mathcal{O}_{4,1}^{H}\left({ }^{3} S_{1}^{[1]}\right)\right| 0\right\rangle$ and $\left\langle 0\left|\mathcal{O}_{4,2}^{H}\left({ }^{3} S_{1}^{[1]}\right)\right| 0\right\rangle$, one can replace $|H+X\rangle\langle H+X|$ in the projector $\mathcal{P}_{H(M, 0)}$ with $|H\rangle\langle H|$ (vacuum-saturation approximation), making an error of relative order $v^{4}$. If one takes this approximation and evaluates the LDMEs in dimensional regularization in a potential model, then they are equal [28]. Since the static potential model is valid up to corrections of order $v^{2}$, we have

$$
\left\langle 0\left|\mathcal{O}_{4,1}^{H}\left({ }^{3} S_{1}^{[1]}\right)\right| 0\right\rangle=\left\langle 0\left|\mathcal{O}_{4,2}^{H}\left({ }^{3} S_{1}^{[1]}\right)\right| 0\right\rangle+\mathcal{O}\left(v^{2}\right) .
$$

Hence, up to corrections of relative order $v^{2}$, only the sum of short-distance coefficients $d_{4,1}\left({ }^{3} S_{1}^{[1]}\right)(z)+d_{4,2}\left({ }^{3} S_{1}^{[1]}\right)(z)$ appears in the fragmentation function.

\subsection{NRQCD factorization formulas for $g \rightarrow J / \psi$ through order $v^{4}$}

In summary, we have the following NRQCD factorization formulas for gluon fragmentation into $J / \psi$ through relative order $v^{4}$.

In relative order $v^{0}$ we have

$$
D_{0}[g \rightarrow J / \psi]=d_{0}\left[g \rightarrow Q \bar{Q}\left({ }^{3} S_{1}^{[1]}\right)\right]\left\langle 0\left|\mathcal{O}_{0}^{J / \psi}\left({ }^{3} S_{1}^{[1]}\right)\right| 0\right\rangle .
$$

The short-distance coefficient $d_{0}\left[g \rightarrow Q \bar{Q}\left({ }^{3} S_{1}^{[1]}\right)\right]$ was calculated in Refs. [19, 20].

In relative order $v^{2}$ we have

$$
D_{2}[g \rightarrow J / \psi]=d_{2}\left[g \rightarrow Q \bar{Q}\left({ }^{3} S_{1}^{[1]}\right)\right]\left\langle 0\left|\mathcal{O}_{2}^{J / \psi}\left({ }^{3} S^{[1]}\right)\right| 0\right\rangle .
$$

The short-distance coefficient $d_{2}\left[g \rightarrow Q \bar{Q}\left({ }^{3} S_{1}^{[1]}\right)\right]$ was calculated in Ref. [21].

In relative order $v^{3}$ we have

$$
D_{3}[g \rightarrow J / \psi]=d_{0}\left[g \rightarrow Q \bar{Q}\left({ }^{1} S_{0}^{[8]}\right)\right]\left\langle 0\left|\mathcal{O}_{0}^{J / \psi}\left({ }^{1} S_{0}^{[8]}\right)\right| 0\right\rangle .
$$

The short-distance coefficient $d_{0}\left[g \rightarrow Q \bar{Q}\left({ }^{1} S_{0}^{[1]}\right)\right]$ was calculated in Ref. [29] and differs from the short-distance coefficient $d_{0}\left[g \rightarrow Q \bar{Q}\left({ }^{1} S_{0}^{[8]}\right)\right]$ in Eq. (3.13) only by a color factor, which we provide in Sec. 7 .

In relative order $v^{4}$ we have

$$
\begin{aligned}
D_{4}[g \rightarrow J / \psi] & =\left\{d_{4,1}\left[g \rightarrow Q \bar{Q}\left({ }^{3} S_{1}^{[1]}\right)\right]+d_{4,2}\left[g \rightarrow Q \bar{Q}\left({ }^{3} S_{1}^{[1]}\right)\right]\right\}\left\langle 0\left|\mathcal{O}_{4}^{J / \psi}\left({ }^{3} S_{1}^{[1]}\right)\right| 0\right\rangle \\
& +d_{0}\left[g \rightarrow Q \bar{Q}\left({ }^{3} P^{[8]}\right)\right]\left\langle 0\left|\mathcal{O}_{0}^{J / \psi}\left({ }^{3} P^{[8]}\right)\right| 0\right\rangle \\
& +d_{0}\left[g \rightarrow Q \bar{Q}\left({ }^{3} S_{1}^{[8]}\right)\right]\left\langle 0\left|\mathcal{O}_{0}^{J / \psi}\left({ }^{3} S_{1}^{[8]}\right)\right| 0\right\rangle
\end{aligned}
$$

The short-distance coefficient $d_{0}\left[g \rightarrow Q \bar{Q}\left({ }^{3} S_{1}^{[8]}\right)\right]$ was calculated at LO in $\alpha_{s}$ in Refs. [21, 29] and at NLO in $\alpha_{s}$ in Refs. [30, 31]. We verify the LO calculations in Refs. [21, 29] in the present paper, giving our result in Sec. 7. We compute $d_{0}\left[g \rightarrow Q \bar{Q}\left({ }^{3} P^{[8]}\right)\right]$ in this paper, giving the result in Sec. 7. The short-distance coefficient $d_{0}\left[g \rightarrow Q \bar{Q}\left({ }^{3} P^{[1]}\right)\right]$ was calculated in Refs. [29] and differs from the short-distance coefficient $d_{0}\left[g \rightarrow Q \bar{Q}\left({ }^{3} P^{[8]}\right)\right]$ in Eq. (3.14) only by a color factor. The computation of the combination of short-distance coefficients $d_{4,1}\left[g \rightarrow Q \bar{Q}\left({ }^{3} S_{1}^{[1]}\right)\right]+d_{4,2}\left[g \rightarrow Q \bar{Q}\left({ }^{3} S_{1}^{[1]}\right)\right]$ is the main goal of this paper. The result of that computation is given in Sec. 7 . 


\section{Kinematics}

In the calculations to follow, in both full QCD and NRQCD, we employ the following kinematics.

We take the $Q$ and the $\bar{Q}$ to be free (on-shell) states with momenta

$$
\begin{aligned}
& p=\frac{1}{2} P+q, \\
& \bar{p}=\frac{1}{2} P-q,
\end{aligned}
$$

respectively. The heavy quark has three-momentum $\boldsymbol{q}$ in the $Q \bar{Q}$ rest frame, and, so, the invariant mass of the $Q \bar{Q}$ state is

$$
P^{2}=M^{2}=4 E^{2},
$$

where

$$
E=\sqrt{m^{2}+\boldsymbol{q}^{2}} .
$$

We work in the frame in which the transverse momentum of the $Q \bar{Q}$ pair vanishes. In this frame, the initial-state gluon, the final-state $Q \bar{Q}$ pair and the final-state gluons, respectively, have the momenta

$$
\begin{aligned}
k & =\left(k^{+}, k^{-}=\frac{k^{2}+\left(P_{\perp} / z\right)^{2}}{2 k^{+}},-\frac{\boldsymbol{P}_{\perp}}{z}\right), \\
P & =\left(z k^{+}, \frac{M^{2}}{2 z k^{+}}, \mathbf{0}_{\perp}\right), \\
k_{1} & =\left(z_{1} k^{+}, \frac{k_{1 \perp}^{2}}{2 z_{1} k^{+}}, \boldsymbol{k}_{1 \perp}\right), \\
k_{2} & =\left(z_{2} k^{+}, \frac{k_{2 \perp}^{2}}{2 z_{2} k^{+}}, \boldsymbol{k}_{2 \perp}\right),
\end{aligned}
$$

where we have introduced the longitudinal momentum fractions

$$
\begin{aligned}
z & =\frac{P^{+}}{k^{+}}, \\
z_{1} & =\frac{k_{1}^{+}}{k^{+}}, \\
z_{2} & =\frac{k_{2}^{+}}{k^{+}} .
\end{aligned}
$$

Because of the conservation of four-momentum, $k=P+k_{1}+k_{2}$, the momenta $k, k_{1}$ and $k_{2}$ depend implicitly on $P$, and, therefore, on $q$. We can make the dependence on $q$ explicit by writing quantities in terms of dimensionless momenta

$$
\begin{aligned}
\bar{P} & =\frac{P}{\sqrt{P^{2}}}, \\
\bar{k} & =\frac{k}{\sqrt{P^{2}}}, \\
\bar{k}_{1} & =\frac{k_{1}}{\sqrt{P^{2}}}, \\
\bar{k}_{2} & =\frac{k_{2}}{\sqrt{P^{2}}} .
\end{aligned}
$$


It is also useful to express the Lorentz invariants in terms of the following dimensionless variables:

$$
\begin{aligned}
e_{1} & =\bar{k}_{1} \cdot \bar{P} \\
e_{2} & =\bar{k}_{2} \cdot \bar{P} \\
x & =\bar{k}_{1} \cdot \bar{k}_{2}=e_{1} e_{2}\left(1-\hat{\boldsymbol{k}}_{1} \cdot \hat{\boldsymbol{k}}_{2}\right),
\end{aligned}
$$

where $\hat{\boldsymbol{k}}_{i}$ is the unit vector that is parallel to the three-vector $\boldsymbol{k}_{i}$ in the $Q \bar{Q}$ rest frame.

The phase space in Eq. (2.7) can be expressed in terms of the dimensionless variables as

$$
\begin{aligned}
& d \Phi_{0}=\frac{4 \pi M}{k^{+}} \delta(1-z) \\
& d \Phi_{1}=\frac{4 \pi M^{d-1}}{k^{+}} \theta\left(z_{1}\right) \delta\left(1-z-z_{1}\right) \frac{d z_{1}}{4 \pi z_{1}} \frac{d^{d-2} \overline{\boldsymbol{k}}_{1 \perp}}{(2 \pi)^{d-2}} \\
& d \Phi_{2}=\frac{4 \pi M^{2 d-3}}{S k^{+}} \theta\left(z_{1}\right) \theta\left(z_{2}\right) \delta\left(1-z-z_{1}-z_{2}\right) \frac{d z_{1}}{4 \pi z_{1}} \frac{d z_{2}}{4 \pi z_{2}} \frac{d^{d-2} \overline{\boldsymbol{k}}_{1 \perp}}{(2 \pi)^{d-2}} \frac{d^{d-2} \overline{\boldsymbol{k}}_{2 \perp}}{(2 \pi)^{d-2}}
\end{aligned}
$$

We have not replaced the overall factor $1 / k^{+}$in Eq. (4.8) with $1 /\left(\bar{k}^{+} \sqrt{P^{2}}\right)$, because it ultimately will be cancelled by the factor $k^{+}$in $C_{\text {frag }}$ in Eq. (2.5). The ranges of the variables $z, z_{1}$ and $z_{2}$ are completely determined by the $\delta$ and $\theta$ functions. When we expand the fragmentation function in powers of $q$, it is convenient to make use of the phase space at LO in $q$,

$$
d \tilde{\Phi}_{n}=\left.d \Phi_{n}\right|_{\boldsymbol{q} \rightarrow \mathbf{0}},
$$

where $\boldsymbol{q} \rightarrow \mathbf{0}$ means that, in the phase space in Eq. (4.8), we replace $M$ with $2 m$. Then $d \Phi_{n}$ can be expressed in terms of $d \tilde{\Phi}_{n}$ as follows:

$$
\begin{aligned}
d \Phi_{0} & =\frac{E}{m} d \tilde{\Phi}_{0}, \\
d \Phi_{1} & =\left(\frac{E}{m}\right)^{3-2 \epsilon} d \tilde{\Phi}_{1}, \\
d \Phi_{2} & =\left(\frac{E}{m}\right)^{5-4 \epsilon} d \tilde{\Phi}_{2} .
\end{aligned}
$$

We express our results for the fragmentation contributions in terms of integrals over the phase spaces $d \tilde{\Phi}_{n}$ :

$$
\tilde{D}[g \rightarrow Q \bar{Q}]=\left.D[g \rightarrow Q \bar{Q}]\right|_{d \Phi_{n} \rightarrow d \tilde{\Phi}_{n}} .
$$

The factors of $E / m$ in Eq. (4.10) are then an additional source of relativistic corrections.

\section{$5 \quad$ Full-QCD calculations}

In this section, we compute the relevant fragmentation functions for free $Q \bar{Q}$ states in full QCD. We have carried out the calculations by writing independent codes using REDUCE [32] and using the FEYNCALC package [33] in MATHEMATica [34]. At each stage of the calculations we have checked that the independent codes give identical results. 
The computations are carried out in $d=4-2 \epsilon$ dimensions with dimensional-regularization scale $\mu$. We use the modified-minimal-subtraction $(\overline{\mathrm{MS}})$ scheme throughout. Then, in $d=4-2 \epsilon$ dimensions, there is a factor $\left[\mu^{2} \exp \left(\gamma_{\mathrm{E}}\right) /(4 \pi)\right]^{\epsilon}$ that is associated with each factor of the strong coupling $\alpha_{s}$, where $\gamma_{\mathrm{E}}$ is the Euler-Mascheroni constant.

In computing the $Q \bar{Q}$ fragmentation functions, it is convenient to make use of projection operators for the spin and color states of the $Q \bar{Q}$ pair. The projection operators for a $Q \bar{Q}$ pair in the color-singlet and color-octet configurations are

$$
\begin{aligned}
\Lambda_{1} & =\frac{1}{\sqrt{N_{c}}} \mathbb{1}, \\
\Lambda_{8}^{a} & =\sqrt{2} T^{a},
\end{aligned}
$$

where $\mathbb{1}$ and $T^{a}$ are the identity matrix and the generator of the fundamental (triplet) representation of $\mathrm{SU}(3), a$ is the adjoint-representation color index $\left(a=1, \ldots, N_{c}^{2}-1\right)$, and $N_{c}=3$. Spin-projection operators at LO in $v$ were first given in Refs. [35-39]. Projectors accurate to all orders in $v$ were given in Ref. [26]. For the spin-triplet state, the projection operator, correct to all orders in $v$, is

$$
\Lambda\left(P, q, \epsilon_{S}^{*}\right)=N(\not{p}-m) \notin_{S}^{*} \frac{P+2 E}{4 E}(\not p+m),
$$

where $\epsilon_{S}$ is the spin polarization of the $Q \bar{Q}$ pair, and $N=[2 \sqrt{2} E(E+m)]^{-1}$. Note that we use nonrelativistic normalization for the heavy-quark spinors.

The use of the spin projection (5.2) in $d$ dimensions requires some justification. It accounts for only the $d-1$ vector polarization states, which, in the $Q \bar{Q}$ rest frame, correspond to the $d-1$ Pauli matrices $\sigma_{i}$. In general, in $d$ dimensions, one must consider states that correspond to products of the $\sigma_{i}$ that are linearly independent of the $\sigma_{i}$ [29]. These additional states vanish as $\epsilon$ goes to zero. Hence, they can contribute only in conjunction with a pole in $\epsilon$. The poles in $\epsilon$ in our calculation correspond to soft divergences. The divergent parts of soft interactions arise from the convection current on fermions lines, and, hence, do not change the fermion spin. Therefore, the additional states that correspond to products of the $\sigma_{i}$ never mix in our calculation with the vector states that correspond to the $\sigma_{i}$. Consequently, we need consider only the $d-1$ vector states in our calculation. ${ }^{2}$

The spin-triplet, color-singlet part of an amplitude $\mathcal{C}$ is

$$
\mathcal{M}=\operatorname{Tr}\left[\mathcal{C}\left(\Lambda \otimes \Lambda_{1}\right)\right]
$$

and the spin-triplet, color-octet part of an amplitude $\mathcal{C}$ is

$$
\mathcal{M}^{a}=\operatorname{Tr}\left[\mathcal{C}\left(\Lambda \otimes \Lambda_{8}^{a}\right)\right],
$$

where the traces are over the Dirac and color indices. The amplitude $\mathcal{C}$ includes the propagator of the initial gluon, as well as the associated polarization factor in Eq. (2.6). In our calculation, the amplitudes $\mathcal{C}, \mathcal{M}$, and $\mathcal{M}^{a}$ are all expressed in terms of the dimensionless

\footnotetext{
${ }^{2}$ Some elements of this argument were presented in Ref. [40].
} 
variables in Eq. (4.6) or invariants that are formed from them, and so the dependence on $q$ is explicit.

The $S$-wave part of $\mathcal{M}$ (with color index suppressed in the color-octet case) can be written as an expansion in powers of $v^{2}=\boldsymbol{q}^{2} / m^{2}$ :

$$
\mathcal{M}_{S}=\mathcal{M}_{S 0}+\mathcal{M}_{S 2}+\mathcal{M}_{S 4}+O\left(\boldsymbol{q}^{6} / m^{6}\right)
$$

where

$$
\begin{aligned}
& \mathcal{M}_{S 0}=(\mathcal{M})_{\boldsymbol{q} \rightarrow \mathbf{0}}, \\
& \mathcal{M}_{S 2}=\frac{\boldsymbol{q}^{2}}{2 !(d-1)} I^{\alpha \beta}\left(\frac{\partial^{2} \mathcal{M}}{\partial q^{\alpha} \partial q^{\beta}}\right)_{\boldsymbol{q} \rightarrow \mathbf{0}}, \\
& \mathcal{M}_{S 4}=\frac{\boldsymbol{q}^{4}}{4 !(d-1)(d+1)} I^{\alpha \beta \gamma \delta}\left(\frac{\partial^{4} \mathcal{M}}{\partial q^{\alpha} \partial q^{\beta} \partial q^{\gamma} \partial q^{\delta}}\right)_{\boldsymbol{q} \rightarrow \mathbf{0}},
\end{aligned}
$$

and

$$
\begin{aligned}
I^{\alpha \beta} & =-g^{\alpha \beta}+P^{\alpha} P^{\beta} /\left(4 E^{2}\right), \\
I^{\alpha \beta \gamma \delta} & =I^{\alpha \beta} I^{\gamma \delta}+I^{\alpha \gamma} I^{\beta \delta}+I^{\alpha \delta} I^{\beta \gamma} .
\end{aligned}
$$

In order to project out the $P$-wave part of the amplitude $\mathcal{M}$, we multiply $\mathcal{M}$ by the $P$ wave orbital-angular-momentum state $-\sqrt{d-1} \epsilon_{L}^{*} \cdot \hat{q}$ and average over the direction of $\boldsymbol{q}^{3}$ Here, $\epsilon_{L}$ is the polarization vector for the orbital-angular-momentum state, and $\hat{q}=(0, \hat{\boldsymbol{q}})$ in the rest frame of the $Q \bar{Q}$ pair. Then, the $P$-wave part of the amplitude is

$$
\mathcal{M}_{P}=\mathcal{M}_{P 1}+O\left(\boldsymbol{q}^{3} / m^{3}\right)
$$

where

$$
\mathcal{M}_{P 1}=-\frac{|\boldsymbol{q}|}{\sqrt{d-1}} \epsilon_{L \alpha}^{*} I^{\alpha \beta}\left(\frac{\partial \mathcal{M}}{\partial q^{\beta}}\right)_{\boldsymbol{q} \rightarrow \mathbf{0}} .
$$

We define squared amplitudes for the color-singlet and color-octet states as

$$
\begin{aligned}
& \mathcal{A}\left({ }^{2 s+1} l_{j}^{[1]}\right)=C_{\text {frag }}\left|\mathcal{M}\left({ }^{2 s+1} l_{j}^{[1]}\right)\right|^{2}, \\
& \mathcal{A}\left({ }^{2 s+1} l_{j}^{[8]}\right)=C_{\text {frag }} \sum_{a}\left|\mathcal{M}^{a}\left({ }^{2 s+1} l_{j}^{[8]}\right)\right|^{2},
\end{aligned}
$$

where $C_{\text {frag }}$ is given in Eq. (2.5), and it is implicit that there are sums over the spin and orbital-angular-momentum polarizations of the $Q \bar{Q}$ states and sums over the polarizations of the initial and final gluons. Note that

$$
\sum_{\lambda} \epsilon_{S}^{\alpha *}(\lambda) \epsilon_{S}^{\beta}(\lambda)=\sum_{\lambda} \epsilon_{L}^{\alpha *}(\lambda) \epsilon_{L}^{\beta}(\lambda)=I^{\alpha \beta}
$$

We denote the order- $v^{k}$ contribution to $\mathcal{A}$ by $\mathcal{A}_{k}$. 


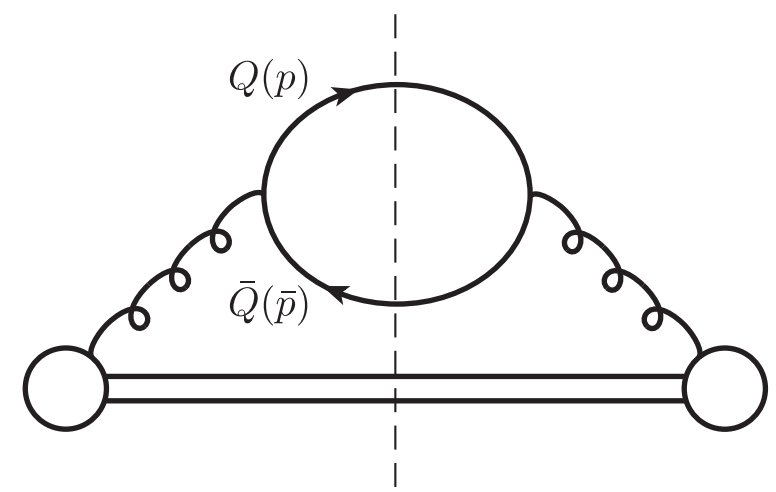

Figure 2. Feynman diagram for the fragmentation process $g \rightarrow Q \bar{Q}\left({ }^{3} S_{1}^{[8]}\right)$. The dashed line represents the final-state cut. The momenta for $Q$ and $\bar{Q}$ on the left side of the cut are $p=\frac{1}{2} P+q$ and $\bar{p}=\frac{1}{2} P-q$, respectively. The momenta on the right side of the cut are $\frac{1}{2} P+q^{\prime}$ and $\frac{1}{2} P-q^{\prime}$, respectively. Here, $\left|\boldsymbol{q}^{\prime}\right|=|\boldsymbol{q}|$ in the rest frame of the $Q \bar{Q}$ pair, but we distinguish the directions of $\boldsymbol{q}$ and $\boldsymbol{q}^{\prime}$ in order to be able to project out orbital-angular-momentum states in the amplitude and its complex conjugate.

\section{$\mathbf{5 . 1} D_{0}\left[g \rightarrow Q \bar{Q}\left({ }^{3} S_{1}^{[8]}\right)\right]$}

The diagram for gluon fragmentation into a ${ }^{3} S_{1}$ color-octet $Q \bar{Q}$ pair at order $v^{0}$ and $\alpha_{s}^{1}$ is shown in Fig. 2. A straightforward computation yields

$$
\mathcal{A}_{0}\left({ }^{3} S_{1}^{[8]}\right)=\frac{\alpha_{s} k^{+}}{8 m^{4}}\left(\frac{\mu^{2}}{4 \pi} e^{\gamma_{\mathrm{E}}}\right)^{\epsilon} .
$$

Carrying out the trivial integration over the phase space $d \tilde{\Phi}_{0}$ in Eqs. (4.8a) and (4.10a), we obtain

$$
D_{0}\left[g \rightarrow Q \bar{Q}\left({ }^{3} S_{1}^{[8]}\right)\right]=\frac{\pi \alpha_{s}}{m^{3}}\left(\frac{\mu^{2}}{4 \pi} e^{\gamma_{\mathrm{E}}}\right)^{\epsilon} \delta(1-z) .
$$

\section{$\mathbf{5 . 2} \quad D_{2}\left[g \rightarrow Q \bar{Q}\left({ }^{3} P^{[8]}\right)\right]$}

The diagrams for gluon fragmentation into a ${ }^{3} P^{[8]}$ color-octet $Q \bar{Q}$ pair at LO in $\alpha_{s}$ and $v$ are shown in Fig. 3. We obtain

$$
\mathcal{A}_{1}\left({ }^{3} P^{[8]}\right)=\frac{k^{+} \pi \alpha_{s}^{2}\left(N_{c}^{2}-4\right) \boldsymbol{q}^{2} z^{1-2 \epsilon}}{8 N_{c}(d-1)(d-2) m^{8}}\left(\frac{\mu^{2}}{4 \pi} e^{\gamma_{\mathrm{E}}}\right)^{2 \epsilon} \sum_{n=0}^{3} \frac{\rho_{n}(z)}{e_{1}^{n}\left(1+2 e_{1}\right)^{2}},
$$

where $\rho_{n}(z)$ are given by

$$
\begin{aligned}
& \rho_{0}(z)=(3-4 \epsilon)\left(2-2 \epsilon-4 z+4 z^{2}\right), \\
& \rho_{1}(z)=2\left[5-10 \epsilon+4 \epsilon^{2}-z(5-12 \epsilon)+2 z^{2}(1-4 \epsilon)\right], \\
& \rho_{2}(z)=3-12 \epsilon+4 \epsilon^{2}+2 z(3+4 \epsilon)-z^{2}(5+4 \epsilon), \\
& \rho_{3}(z)=-2(1-z)^{2} .
\end{aligned}
$$

\footnotetext{
${ }^{3}$ In some calculations in NRQCD, the $P$-wave orbital-angular-momentum state is normalized as $-\epsilon_{L}^{*} \cdot \hat{q}$.
} 


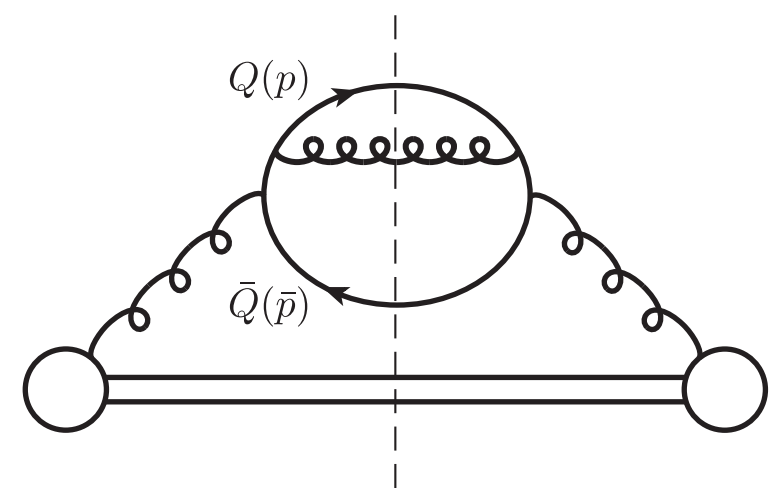

Figure 3. One of the four Feynman diagrams for the fragmentation process $g \rightarrow Q \bar{Q}\left({ }^{3} P^{[8]}\right)$. Three additional diagrams can be obtained by permuting the gluon-fermion vertices on the left and right sides of the cut.

We carry out the integration over the phase space $d \tilde{\Phi}_{1}$ in Eqs. (4.8b) and (4.10b) by making use of the methods that are described in Appendix A. Then, we obtain

$$
\begin{aligned}
D_{2}\left[g \rightarrow Q \bar{Q}\left({ }^{3} P^{[8]}\right)\right]= & \frac{8 \alpha_{s}^{2} \boldsymbol{q}^{2}}{(d-1) m^{5}} \frac{N_{c}^{2}-4}{4 N_{c}}(1-\epsilon) \Gamma(1+\epsilon)\left(\frac{\mu^{2}}{4 \pi} e^{\gamma_{\mathrm{E}}}\right)^{\epsilon}\left(\frac{\mu^{2}}{4 m^{2}} e^{\gamma_{\mathrm{E}}}\right)^{\epsilon} \\
& \times\left[-\frac{1}{2 \epsilon_{\mathrm{IR}}} \delta(1-z)+f(z)\right]
\end{aligned}
$$

where the finite function $f(z)$ is defined by

$$
\begin{aligned}
f(z)= & {\left[\frac{1}{(1-z)^{1+2 \epsilon}}\right]_{+}+\frac{1}{4(1-\epsilon)^{2}}\left\{\frac{(1-z)^{-\epsilon}-(1-z)^{-2 \epsilon}}{\epsilon}(13-7 z)\right.} \\
& +(1-z)^{-2 \epsilon}\left[10+3 z-5 z^{2}+2 \epsilon\left(2-2 z+z^{2}\right)-4 \epsilon^{2}\right] \\
& \left.+\frac{1}{2}(1-z)^{-\epsilon}\left[-28+15 z+\epsilon(8-11 z)+4 \epsilon^{2} z\right]\right\} .
\end{aligned}
$$

Here, the distribution $[g(z)]_{+}$is defined by

$$
\int_{0}^{1} d z h(z)[g(z)]_{+} \equiv \int_{0}^{1} d z[h(z)-h(1)] g(z) .
$$

In extracting the pole in Eq. (5.16), we have made use of the identity,

$$
\frac{1}{(1-z)^{1+n \epsilon}}=-\frac{1}{n \epsilon} \delta(1-z)+\left[\frac{1}{(1-z)^{1+n \epsilon}}\right]_{+},
$$

which applies when the domain of integration is $0 \leq z \leq 1$. The expression in Eq. (5.16b) gives the exact $\epsilon$ dependence. We can expand the plus function $\left[1 /(1-z)^{1+n \epsilon}\right]_{+}$as

$$
\left[\frac{1}{(1-z)^{1+n \epsilon}}\right]_{+}=\sum_{k=0}^{\infty} \frac{(-n \epsilon)^{k}}{k !}\left[\frac{\log ^{k}(1-z)}{1-z}\right]_{+} .
$$




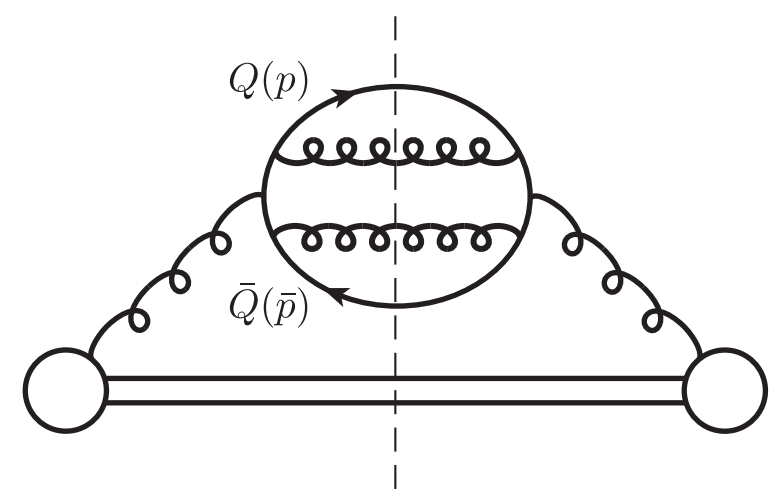

Figure 4. One of the 36 Feynman diagrams for the fragmentation process $g \rightarrow Q \bar{Q}\left({ }^{3} S_{1}^{[1]}\right)$. Thirtyfive additional diagrams can be obtained by permuting the gluon-fermion vertices on the left and right sides of the cut.

In the analysis of $f(z)$, we need to keep only terms through order $\epsilon^{1}$. Then, we can simplify $f(z)$ as follows:

$$
\begin{aligned}
f(z) & =\left(\frac{1}{1-z}\right)_{+}-2 \epsilon\left[\frac{\log (1-z)}{1-z}\right]_{+}+\frac{1}{8}\left[-8+21 z-10 z^{2}+2(13-7 z) \log (1-z)\right] \\
& +\frac{\epsilon}{8}\left[(23-16 z) z+5\left(8-11 z+4 z^{2}\right) \log (1-z)-3(13-7 z) \log ^{2}(1-z)\right]+O\left(\epsilon^{2}\right) .
\end{aligned}
$$

\section{3 $D_{4}\left[g \rightarrow Q \bar{Q}\left({ }^{3} S_{1}^{[1]}\right)\right]$}

The diagrams for gluon fragmentation into a ${ }^{3} S_{1}$ color-singlet $Q \bar{Q}$ pair at LO in $\alpha_{s}$ are shown in Fig. 4. Through relative order $v^{4}$, the relevant squared amplitudes are

$$
\begin{aligned}
& \mathcal{A}_{0}\left({ }^{3} S_{1}^{[1]}\right)=C_{\text {frag }}\left|\mathcal{M}_{0}\left({ }^{3} S_{1}^{[1]}\right)\right|^{2}, \\
& \mathcal{A}_{2}\left({ }^{3} S_{1}^{[1]}\right)=2 C_{\text {frag }} \operatorname{Re}\left[\mathcal{M}_{2}\left({ }^{3} S_{1}^{[1]}\right) \mathcal{M}_{0}^{*}\left({ }^{3} S_{1}^{[1]}\right)\right], \\
& \mathcal{A}_{4}\left({ }^{3} S_{1}^{[1]}\right)=C_{\text {frag }}\left\{\left|\mathcal{M}_{2}\left({ }^{3} S_{1}^{[1]}\right)\right|^{2}+2 \operatorname{Re}\left[\mathcal{M}_{4}\left({ }^{3} S_{1}^{[1]}\right) \mathcal{M}_{0}^{*}\left({ }^{3} S_{1}^{[1]}\right)\right]\right\} .
\end{aligned}
$$

The order- $v^{0}$ contribution $\mathcal{A}_{0}\left({ }^{3} S_{1}^{[1]}\right)$ and the order- $v^{2}$ contribution $\mathcal{A}_{2}\left({ }^{3} S_{1}^{[1]}\right)$ have been computed previously in Ref. [21]. Here, we wish to compute the order- $v^{4}$ contribution $\mathcal{A}_{4}\left({ }^{3} S_{1}^{[1]}\right)$.

The integration of $\mathcal{A}_{4}\left({ }^{3} S_{1}^{[1]}\right)$ over the phase space contains soft divergences. These can arise when one or both of the final-state gluons become soft. We identify the divergent part of the integrand in Eq. (5.21c) that arises when both gluons become soft by making the substitutions $k_{1} \rightarrow k_{1} \lambda$ and $k_{2} \rightarrow k_{2} \lambda$, multiplying by $\lambda^{4}$, and taking the limit $\lambda \rightarrow 0$. The result is

$$
\mathcal{S}_{12}=\frac{C_{\mathcal{S}}(d-2)}{e_{1}^{4} e_{2}^{4}}\left[(d-2) e_{1}^{2} e_{2}^{2}-2 e_{1} e_{2} x+x^{2}\right]
$$

where

$$
C_{\mathcal{S}}=\frac{k^{+} \pi^{2} \alpha_{s}^{3} z^{d-3}}{(d-1)^{2}(d-2) m^{8}} \frac{N_{c}^{2}-4}{4 N_{c}^{2}}\left(\frac{\mu^{2}}{4 \pi} e^{\gamma_{\mathrm{E}}}\right)^{3 \epsilon} \frac{\boldsymbol{q}^{4}}{m^{4}}
$$


We identify the divergent part of the integrand in Eq. (5.21c) that arises when only $k_{1}$ $\left(k_{2}\right)$ becomes soft by subtracting $\mathcal{S}_{12}$, making the substitution $k_{1} \rightarrow k_{1} \lambda\left(k_{2} \rightarrow k_{2} \lambda\right)$, multiplying by $\lambda^{2}$, and taking the limit $\lambda \rightarrow 0$. The result is

$$
\begin{aligned}
& \mathcal{S}_{1}=\frac{C_{\mathcal{S}}}{e_{1}^{4} e_{2}^{4}\left(1+2 e_{2}\right)^{2}} \sum_{n=0}^{2} x^{n} h_{n}\left(e_{1}, e_{2}, z_{1}, z_{2}\right), \\
& \mathcal{S}_{2}=\frac{C_{\mathcal{S}}}{e_{2}^{4} e_{1}^{4}\left(1+2 e_{1}\right)^{2}} \sum_{n=0}^{2} x^{n} h_{n}\left(e_{2}, e_{1}, z_{2}, z_{1}\right),
\end{aligned}
$$

where the $h_{n}\left(e_{1}, e_{2}, z_{1}, z_{2}\right)$ are given by

$$
\begin{aligned}
h_{0}\left(e_{1}, e_{2}, z_{1}, z_{2}\right)= & 2 e_{2}\left(1+2 e_{2}\right)\left\{-2(3-2 \epsilon)(1-\epsilon) e_{1}^{2} e_{2}^{2}+z_{1} e_{1} e_{2}^{2}+z_{2} e_{1}^{2} e_{2}\right. \\
& \times\left[1-2(1-2 \epsilon) e_{2}\right]-z_{1}^{2} e_{2}^{2}\left(1+\epsilon e_{2}\right)+z_{1} z_{2} e_{1} e_{2}\left[1-2(1-\epsilon) e_{2}\right] \\
& \left.+z_{2}^{2} e_{1}^{2}\left[-1+(1-3 \epsilon) e_{2}+2(1-2 \epsilon) e_{2}^{2}\right]\right\}+4(3-2 \epsilon)(1-\epsilon) e_{1}^{2} e_{2}^{4},
\end{aligned}
$$

$$
\begin{aligned}
h_{1}\left(e_{1}, e_{2}, z_{1}, z_{2}\right)= & 2 e_{2}\left(1+2 e_{2}\right)\left\{z_{1} e_{2}\left(1+2 \epsilon e_{2}\right)+z_{2} e_{1}\left[1+(3-2 \epsilon) e_{2}\right]-z_{1} z_{2}\left(1+2 \epsilon e_{2}\right.\right. \\
& \left.\left.+2 \epsilon e_{2}^{2}\right)-z_{2}^{2} e_{1}\left[3-2 \epsilon+2(1-\epsilon) e_{2}\right]\right\}+2 e_{1} e_{2}^{2}\left[5-4 \epsilon+6(1-\epsilon) e_{2}\right],
\end{aligned}
$$

$$
\begin{aligned}
h_{2}\left(e_{1}, e_{2}, z_{1}, z_{2}\right)= & -\left(1+2 e_{2}\right)\left\{e_{2}\left(4-3 \epsilon+2 \epsilon e_{2}\right)+2 z_{2} e_{2}\left(1-2 \epsilon-2 \epsilon e_{2}\right)\right. \\
& \left.-2 z_{2}^{2}\left(1+e_{2}\right)\left(1-\epsilon-\epsilon e_{2}\right)\right\}-(2-\epsilon) e_{2} .
\end{aligned}
$$

We carry out the integrations of $\mathcal{S}_{12}, \mathcal{S}_{1}$ and $\mathcal{S}_{2}$ over the phase space $d \tilde{\Phi}_{2}$ in Eq. (4.10c) [see also Eq. (4.8c)] by making use of the methods that are described in Appendix A. Then, we obtain

$$
\begin{aligned}
I\left[\mathcal{S}_{12}\right]= & \left\{\frac{1}{8 \epsilon_{\mathrm{IR}}^{2}} \delta(1-z)-\frac{1}{2 \epsilon_{\mathrm{IR}}}\left[\frac{1}{(1-z)^{1+4 \epsilon}}\right]_{+}+\frac{1-z^{1+2 \epsilon}}{2 \epsilon_{\mathrm{IR}}(1-z)^{1+4 \epsilon}}\right\} \\
& \times\left(\frac{8 \alpha_{s}}{3 \pi m^{2}}\right)^{2} \frac{N_{c}^{2}-4}{16 N_{c}^{2}} \frac{\pi \alpha_{s}}{(d-1) m^{3}}\left(\frac{\mu^{2}}{4 \pi} e^{\gamma_{\mathrm{E}}}\right)^{\epsilon} \frac{\boldsymbol{q}^{4}}{d-1} \\
& \times\left(\frac{\mu^{2}}{4 m^{2}} e^{\gamma_{\mathrm{E}}}\right)^{2 \epsilon} \frac{\Gamma^{2}(1+\epsilon) \Gamma^{2}(1-2 \epsilon)}{\Gamma(1-4 \epsilon)}(1-\epsilon)\left(6-2 \epsilon-\epsilon^{2}-2 \epsilon^{3}\right),
\end{aligned}
$$

where we have used the identity in Eq. (5.18). If we expand Eq. (5.26) in powers of $\epsilon$, then we find that

$$
\begin{aligned}
I\left[\mathcal{S}_{12}\right]= & \left\{\frac{1}{8 \epsilon_{\mathrm{IR}}^{2}} \delta(1-z)-\frac{1}{2 \epsilon_{\mathrm{IR}}}\left[\delta(1-z)\left(\frac{1}{3}-\log \frac{\mu}{2 m}\right)+\left(\frac{1}{1-z}\right)_{+}-1\right]\right. \\
& +\delta(1-z)\left(\frac{1-3 \pi^{2}}{48}-\frac{2}{3} \log \frac{\mu}{2 m}+\log ^{2} \frac{\mu}{2 m}\right)+\left(\frac{1}{1-z}\right)_{+}\left(\frac{2}{3}-2 \log \frac{\mu}{2 m}\right) \\
& \left.+2\left[\frac{\log (1-z)}{1-z}\right]_{+}-2\left(\frac{1}{3}-\log \frac{\mu}{2 m}\right)-\frac{z \log z}{1-z}-2 \log (1-z)\right\} \\
& \times\left(\frac{8 \alpha_{s}}{3 \pi m^{2}}\right)^{2} \frac{N_{c}^{2}-4}{16 N_{c}^{2}} \frac{\pi \alpha_{s}}{(d-1) m^{3}}\left(\frac{\mu^{2}}{4 \pi} e^{\gamma_{\mathrm{E}}}\right)^{\epsilon} \frac{6 \boldsymbol{q}^{4}}{d-1}+O(\epsilon) .
\end{aligned}
$$


We also find that

$$
\begin{aligned}
I\left[\mathcal{S}_{1}\right] & =I\left[\mathcal{S}_{2}\right]=\left(-\frac{\tau_{1}}{2 \epsilon_{\mathrm{IR}}}+\tau_{0}\right)\left(\frac{\mu^{2}}{4 m^{2}} e^{\gamma_{\mathrm{E}}}\right)^{2 \epsilon} \frac{z^{-2+2 \epsilon}(1-z)^{-4 \epsilon} \Gamma^{2}(1+\epsilon)}{48(1-\epsilon)} \\
& \times\left(\frac{8 \alpha_{s}}{3 \pi m^{2}}\right)^{2} \frac{N_{c}^{2}-4}{16 N_{c}^{2}}\left(\frac{\mu^{2}}{4 \pi} e^{\gamma_{\mathrm{E}}}\right)^{\epsilon} \frac{\pi \alpha_{s}}{(d-1) m^{3}} \frac{\boldsymbol{q}^{4}}{d-1}+O(\epsilon),
\end{aligned}
$$

where

$$
\begin{aligned}
\tau_{0}= & 3\left\{12(13-7 z) z^{2} \operatorname{Li}_{2}(1-z)+3(7 z-13) z^{2} \log ^{2}(1-z)\right. \\
& -2 z\left[z^{3}-\left(43+7 \pi^{2}\right) z^{2}+6(2 z+9) z^{2} \log z+\left(48+13 \pi^{2}\right) z-42\right] \\
& \left.+3\left(8 z^{4}-7 z^{3}+34 z^{2}-44 z+28\right) \log (1-z)\right\}, \\
\tau_{1}= & 18 z^{2}[z(21-10 z)+2(13-7 z) \log (1-z)] .
\end{aligned}
$$

Here, $\operatorname{Li}_{2}(z)$ is the Spence function, which is defined by

$$
\operatorname{Li}_{2}(x)=-\int_{0}^{x} d t \frac{\log (1-t)}{t}=\sum_{k=1}^{\infty} \frac{x^{k}}{k^{2}} .
$$

The fragmentation-function contribution of $\mathcal{A}_{4}\left({ }^{3} S_{1}^{[1]}\right)$ is then

$$
\tilde{D}_{4}\left[g \rightarrow Q \bar{Q}\left({ }^{3} S_{1}^{[1]}\right)\right]=\tilde{D}_{4}\left[g \rightarrow Q \bar{Q}\left({ }^{3} S_{1}^{[1]}\right)\right]^{\text {finite }}+I\left[\mathcal{S}_{12}\right]+I\left[\mathcal{S}_{1}\right]+I\left[\mathcal{S}_{2}\right],
$$

where

$$
\tilde{D}_{4}\left[g \rightarrow Q \bar{Q}\left({ }^{3} S_{1}^{[1]}\right)\right]^{\text {finite }}=\int d \tilde{\Phi}_{2}\left[\mathcal{A}_{4}\left({ }^{3} S_{1}^{[1]}\right)-\mathcal{S}_{12}-\mathcal{S}_{1}-\mathcal{S}_{2}\right] .
$$

Since $\left.\tilde{D}_{4}\left[g \rightarrow Q \bar{Q}\left({ }^{3} S_{1}^{[1]}\right)\right]\right]^{\text {finite }}$ contains no soft divergences, we compute it in $d=4$ dimensions, using numerical integration over the phase space. This computation is described in Sec. 8 .

As was mentioned previously, $D_{4}\left[g \rightarrow Q \bar{Q}\left({ }^{3} S_{1}^{[1]}\right)\right]^{\text {finite }}$ also contains contributions that arise from the difference between the fully relativistic phase space $d \Phi_{2}$ and the order- $v^{0}$ phase space $d \tilde{\Phi}_{2}$ [Eq. (4.10c)]. Hence, we write

$$
D_{4}\left[g \rightarrow Q \bar{Q}\left({ }^{3} S_{1}^{[1]}\right)\right]=D_{4}\left[g \rightarrow Q \bar{Q}\left({ }^{3} S_{1}^{[1]}\right)\right]^{\text {finite }}+I\left[\mathcal{S}_{12}\right]+I\left[\mathcal{S}_{1}\right]+I\left[\mathcal{S}_{2}\right],
$$

where

$$
\begin{aligned}
D_{4}\left[g \rightarrow Q \bar{Q}\left({ }^{3} S_{1}^{[1]}\right)\right]^{\text {finite }}= & \tilde{D}_{4}\left[g \rightarrow Q \bar{Q}\left({ }^{3} S_{1}^{[1]}\right)\right]^{\text {finite }}+\frac{5 \boldsymbol{q}^{2}}{2 m^{2}} \tilde{D}_{2}\left[g \rightarrow Q \bar{Q}\left({ }^{3} S_{1}^{[1]}\right)\right] \\
& +\frac{15 \boldsymbol{q}^{4}}{8 m^{4}} \tilde{D}_{0}\left[g \rightarrow Q \bar{Q}\left({ }^{3} S_{1}^{[1]}\right)\right] \\
\tilde{D}_{0}[g \rightarrow & \left.Q \bar{Q}\left({ }^{3} S_{1}^{[1]}\right)\right]=\int d \tilde{\Phi}_{2} \mathcal{A}_{0}\left({ }^{3} S_{1}^{[1]}\right) \\
& \tilde{D}_{2}\left[g \rightarrow Q \bar{Q}\left({ }^{3} S_{1}^{[1]}\right)\right]=\int d \tilde{\Phi}_{2} \mathcal{A}_{2}\left({ }^{3} S_{1}^{[1]}\right)
\end{aligned}
$$


and we have used the expansion

$$
\left(\frac{E}{m}\right)^{5}=1+\frac{5 \boldsymbol{q}^{2}}{2 m^{2}}+\frac{15 \boldsymbol{q}^{4}}{8 m^{4}}+O\left(\boldsymbol{q}^{6} / m^{6}\right) .
$$

$\tilde{D}_{0}\left[g \rightarrow Q \bar{Q}\left({ }^{3} S_{1}^{[1]}\right)\right]$ and $\tilde{D}_{2}\left[g \rightarrow Q \bar{Q}\left({ }^{3} S_{1}^{[1]}\right)\right]$ are finite, and they have been evaluated in $d=4$ dimensions in Ref. [21]. We have checked those calculations, computing the phase-space integrations in $\tilde{D}_{0}\left[g \rightarrow Q \bar{Q}\left({ }^{3} S_{1}^{[1]}\right)\right]$ and $\tilde{D}_{2}\left[g \rightarrow Q \bar{Q}\left({ }^{3} S_{1}^{[1]}\right)\right]$ numerically.

\section{NRQCD LDMEs}

In this section we compute the NRQCD LDMEs for free $Q \bar{Q}\left({ }^{3} S_{1}^{[1]}\right)$ states that are relevant through relative order $v^{4}$. These computations are carried out in each case at the leading nontrivial order in $\alpha_{s}$ in $d=4-2 \epsilon$ dimensions, with dimensional-regularization scale $\mu$. We remind the reader that, because we use the $\overline{\mathrm{MS}}$ scheme in computing the $\mathrm{QCD}$ corrections to NRQCD LDMEs, there is a factor $\left[\mu^{2} \exp \left(\gamma_{\mathrm{E}}\right) /(4 \pi)\right]^{\epsilon}$ that is associated with each factor of the strong coupling $\alpha_{s}$ in $d$ dimensions.

\subsection{Order $\alpha_{s}^{0}$}

The matrix elements of the $Q \bar{Q}$ NRQCD operators at order $\alpha_{s}^{0}$ are normalized as

$$
\begin{aligned}
& \left\langle 0\left|\mathcal{O}_{0}^{Q \bar{Q}\left({ }^{1} S_{0}^{[8]}\right)}\left({ }^{1} S_{0}^{[8]}\right)\right| 0\right\rangle^{(0)}=\left(N_{c}^{2}-1\right), \\
& \left\langle 0\left|\mathcal{O}_{0}^{\left.Q \bar{Q}^{(3} S_{1}^{[1]}\right)}\left({ }^{3} S_{1}^{[1]}\right)\right| 0\right\rangle^{(0)}=2(d-1) N_{c}, \\
& \left\langle 0\left|\mathcal{O}_{0}^{\left.Q \bar{Q}^{(3} S_{1}^{[8]}\right)}\left({ }^{3} S_{1}^{[8]}\right)\right| 0\right\rangle^{(0)}=(d-1)\left(N_{c}^{2}-1\right), \\
& \left\langle 0\left|\mathcal{O}_{2}^{Q \bar{Q}\left({ }^{3} S_{1}^{[n]}\right)}\left({ }^{3} S_{1}^{[n]}\right)\right| 0\right\rangle^{(0)}=\boldsymbol{q}^{2}\left\langle 0\left|\mathcal{O}_{0}^{Q \bar{Q}\left({ }^{3} S_{1}^{[n]}\right)}\left({ }^{3} S_{1}^{[n]}\right)\right| 0\right\rangle^{(0)}, \\
& \left\langle 0\left|\mathcal{O}_{4}^{Q \bar{Q}\left({ }^{3} S_{1}^{[n]}\right)}\left({ }^{3} S_{1}^{[n]}\right)\right| 0\right\rangle^{(0)}=\boldsymbol{q}^{4}\left\langle 0\left|\mathcal{O}_{0}^{Q \bar{Q}\left({ }^{3} S_{1}^{[n]}\right)}\left({ }^{3} S_{1}^{[n]}\right)\right| 0\right\rangle^{(0)}, \\
& \left\langle 0\left|\mathcal{O}_{0}^{Q \bar{Q}\left({ }^{3} P^{[8]}\right)}\left({ }^{3} P^{[8]}\right)\right| 0\right\rangle^{(0)}=\boldsymbol{q}^{2}(d-1)\left(N_{c}^{2}-1\right),
\end{aligned}
$$

where a sum over the final-state polarizations is implied. The superscript $(k)$ indicates the order in $\alpha_{s}$.

\subsection{Order $\alpha_{s}$}

\subsection{1 ${ }^{3} P^{[8]} \rightarrow{ }^{3} S_{1}^{[1]}$}

In order $\alpha_{s}$, the ${ }^{3} P^{[8]} Q \bar{Q}$ operator can couple to the ${ }^{3} S_{1}^{[1]}$ state through the diagrams that are shown in Fig. $5 .^{4}$

The set of diagrams in Fig. 5 is gauge invariant. We find it convenient to work in the $Q \bar{Q}$ center-of-momentum frame and to compute the diagrams in the Coulomb gauge.

\footnotetext{
${ }^{4}$ We suppress Wilson lines in diagrams involving NRQCD production operators [3, 4] because the diagrams involving interactions with Wilson lines vanish for the orders in $\alpha_{s}$ and the operator and final-state quantum numbers that we consider.
} 


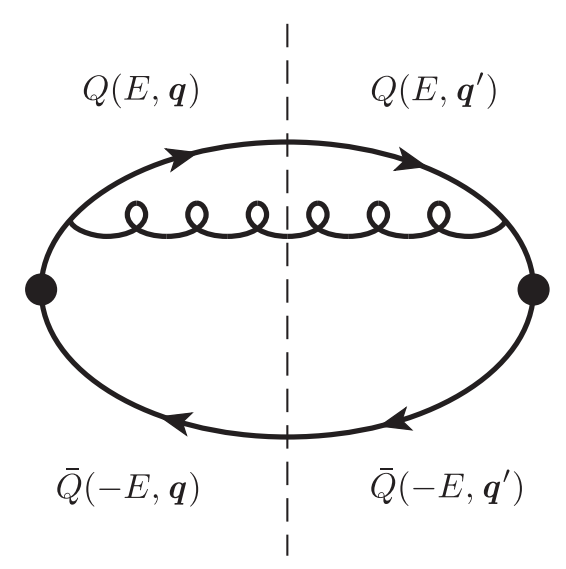

Figure 5. One of the four Feynman diagrams for the computation of the LDME $\left\langle 0\left|\mathcal{O}_{0}^{Q \bar{Q}\left({ }^{3} S_{1}^{[1]}\right)}\left[Q \bar{Q}\left({ }^{3} P^{[8]}\right)\right]\right| 0\right\rangle^{(1)}$. The solid circles represent the $Q \bar{Q}$ operators in the LDME. As in the full-QCD calculation, we take the free $Q$ and $\bar{Q}$ states to have momenta $\frac{1}{2} P+q$ and $\frac{1}{2} P-q$ on the left side of the cut and $\frac{1}{2} P+q^{\prime}$ and $\frac{1}{2} P-q^{\prime}$ on the right side of the cut, where, $\left|\boldsymbol{q}^{\prime}\right|=|\boldsymbol{q}|$ in the rest frame of the $Q \bar{Q}$ pair, but we distinguish the directions of $\boldsymbol{q}$ and $\boldsymbol{q}^{\prime}$ in order to be able to project out orbital-angular-momentum states in the amplitude and its complex conjugate. Three additional diagrams can be obtained by permuting the gluon-fermion and operator vertices on the left and right sides of the cut.

Then, the real gluons in the final state must be transverse. Through relative order $v^{2}$, a straightforward computation in dimensional regularization gives

$$
\left\langle 0\left|\mathcal{O}_{0}^{\left.Q \bar{Q}^{3} S_{1}^{[1]}\right)}\left({ }^{3} P^{[8]}\right)\right| 0\right\rangle^{(1)}=\left(\mathcal{M}_{a}^{(1)}+\mathcal{M}_{b}^{(1)}+\mathcal{M}_{c}^{(1)}+\mathcal{M}_{d}^{(1)}\right)_{{ }^{3} S_{1}^{[1]}},
$$

where

$$
\begin{aligned}
\mathcal{M}_{a}^{(1)}= & \pi \alpha_{s}\left(\frac{\mu^{2}}{4 \pi} e^{\gamma_{\mathrm{E}}}\right)^{\epsilon} \frac{N_{c}^{2}-1}{4 N_{c}^{2}} \xi^{\dagger} \sigma^{k} \eta \eta^{\dagger} \sigma^{k} \xi \\
& \times \int \frac{d^{d-1} \boldsymbol{k}}{2|\boldsymbol{k}|(2 \pi)^{d-1}} \frac{(k+2 q)^{l}(k+2 q)^{i}\left(\delta^{i j}-\hat{\boldsymbol{k}}^{i} \hat{\boldsymbol{k}}^{j}\right)\left(k+2 q^{\prime}\right)^{j}\left(k+2 q^{\prime}\right)^{l}}{\left(\boldsymbol{k}^{2}+2 \boldsymbol{k} \cdot \boldsymbol{q}+2 m|\boldsymbol{k}|\right)\left(\boldsymbol{k}^{2}+2 \boldsymbol{k} \cdot \boldsymbol{q}^{\prime}+2 m|\boldsymbol{k}|\right)}, \\
\mathcal{M}_{b}^{(1)}= & \pi \alpha_{s}\left(\frac{\mu^{2}}{4 \pi} e^{\gamma_{\mathrm{E}}}\right)^{\epsilon} \frac{N_{c}^{2}-1}{4 N_{c}^{2}} \xi^{\dagger} \sigma^{k} \eta \eta^{\dagger} \sigma^{k} \xi \\
& \times \int \frac{d^{d-1} \boldsymbol{k}}{2|\boldsymbol{k}|(2 \pi)^{d-1}} \frac{(-k+2 q)^{l}(-k+2 q)^{i}\left(\delta^{i j}-\hat{\boldsymbol{k}}^{i} \hat{\boldsymbol{k}}^{j}\right)\left(k+2 q^{\prime}\right)^{j}\left(k+2 q^{\prime}\right)^{l}}{\left(\boldsymbol{k}^{2}-2 \boldsymbol{k} \cdot \boldsymbol{q}+2 m|\boldsymbol{k}|\right)\left(\boldsymbol{k}^{2}+2 \boldsymbol{k} \cdot \boldsymbol{q}^{\prime}+2 m|\boldsymbol{k}|\right)}, \\
\mathcal{M}_{c}^{(1)}= & \pi \alpha_{s}\left(\frac{\mu^{2}}{4 \pi} e^{\gamma_{\mathrm{E}}}\right)^{\epsilon} \frac{N_{c}^{2}-1}{4 N_{c}^{2}} \xi^{\dagger} \sigma^{k} \eta \eta^{\dagger} \sigma^{k} \xi \\
& \times \int \frac{d^{d-1} \boldsymbol{k}}{2|\boldsymbol{k}|(2 \pi)^{d-1}} \frac{(-k+2 q)^{l}(-k+2 q)^{i}\left(\delta^{i j}-\hat{\boldsymbol{k}}^{i} \hat{\boldsymbol{k}}^{j}\right)\left(-k+2 q^{\prime}\right)^{j}\left(-k+2 q^{\prime}\right)^{l}}{\left(\boldsymbol{k}^{2}-2 \boldsymbol{k} \cdot \boldsymbol{q}+2 m|\boldsymbol{k}|\right)\left(\boldsymbol{k}^{2}-2 \boldsymbol{k} \cdot \boldsymbol{q}^{\prime}+2 m|\boldsymbol{k}|\right)}, \\
\mathcal{M}_{d}^{(1)}= & \pi \alpha_{s}\left(\frac{\mu^{2}}{4 \pi} e^{\gamma_{\mathrm{E}}}\right)^{\epsilon} \frac{N_{c}^{2}-1}{4 N_{c}^{2}} \xi^{\dagger} \sigma^{k} \eta \eta^{\dagger} \sigma^{k} \xi \\
& \times \int \frac{d^{d-1} \boldsymbol{k}}{2|\boldsymbol{k}|(2 \pi)^{d-1}} \frac{(k+2 q)^{l}(k+2 q)^{i}\left(\delta^{i j}-\hat{\boldsymbol{k}}^{i} \hat{\boldsymbol{k}}^{j}\right)\left(-k+2 q^{\prime}\right)^{j}\left(-k+2 q^{\prime}\right)^{l}}{\left(\boldsymbol{k}^{2}+2 \boldsymbol{k} \cdot \boldsymbol{q}+2 m|\boldsymbol{k}|\right)\left(\boldsymbol{k}^{2}-2 \boldsymbol{k} \cdot \boldsymbol{q}^{\prime}+2 m|\boldsymbol{k}|\right)} .
\end{aligned}
$$


In Eq. (6.3), $\xi$ and $\eta^{\dagger}$ are the Pauli spinors for the free $Q$ and $\bar{Q}$ states, respectively. The subscript ${ }^{3} S_{1}^{[1]}$ in Eq. (6.2) indicates that the bispinors $\xi^{\dagger} \eta$ and $\eta^{\dagger} \xi$ are in color-singlet, spin-triplet states and that we project onto $S$-wave states by averaging over the directions of $\boldsymbol{q}$ and $\boldsymbol{q}^{\prime}$. A sum over the polarizations of the spin-triplet $Q \bar{Q}$ pair is implicit.

We expand the integrands in Eq. (6.3) in powers of $1 / \mathrm{m}$. In dimensional regularization, only the leading power contributes because the expressions for higher powers in $1 / \mathrm{m}$ produce power-divergent, homogeneous integrals. ${ }^{5}$ The result is

$$
\begin{aligned}
\left\langle 0\left|\mathcal{O}_{0}^{Q \bar{Q}^{3}{ }^{[3}[1]}\left({ }^{3} P^{[8]}\right)\right| 0\right\rangle^{(1)}= & \frac{8 \pi \alpha_{s}}{m^{2}} \frac{d-2}{d-1}\left(\frac{\mu^{2}}{4 \pi} e^{\gamma_{\mathrm{E}}}\right)^{\epsilon} \frac{N_{c}^{2}-1}{4 N_{c}^{2}}\left(\xi^{\dagger} q^{\prime i} q^{\prime l} \sigma^{k} \eta \eta^{\dagger} q^{i} q^{l} \sigma^{k} \xi\right)_{{ }^{3} S_{1}^{[1]}} \\
& \times \int \frac{d^{d-1} \boldsymbol{k}}{|\boldsymbol{k}|^{3}(2 \pi)^{d-1}} .
\end{aligned}
$$

The remaining integration over the spatial components of $k$ is straightforward and yields

$$
\left\langle 0\left|\mathcal{O}_{0}^{Q \bar{Q}\left({ }^{3} S_{1}^{[1]}\right)}\left({ }^{3} P^{[8]}\right)\right| 0\right\rangle^{(1)}=\frac{8 \alpha_{s} c(\epsilon)}{3 \pi m^{2}}\left(\frac{1}{2 \epsilon_{\mathrm{UV}}}-\frac{1}{2 \epsilon_{\mathrm{IR}}}\right) \frac{N_{c}^{2}-1}{4 N_{c}^{2}}\left(\xi^{\dagger} q^{\prime i} q^{\prime l} \sigma^{k} \eta \eta^{\dagger} q^{i} q^{l} \sigma^{k} \xi\right)_{{ }_{3} S_{1}^{[1]}} .
$$

Here, we have separated the ultraviolet (UV) and infrared (IR) divergent contributions of the scaleless integral. The quantity $c(\epsilon)$ is given by

$$
c(\epsilon)=\frac{\left(\mu^{2} e^{\gamma_{\mathrm{E}}}\right)^{\epsilon}(1-\epsilon) \Gamma\left(\frac{1}{2}\right)}{\left(1-\frac{2}{3} \epsilon\right)(1-2 \epsilon) \Gamma\left(\frac{1}{2}-\epsilon\right)} .
$$

Note that

$$
c(0)=1
$$

Now,

$$
\begin{aligned}
& \frac{c(\epsilon)}{\epsilon_{\mathrm{UV}}}=\frac{1}{\epsilon_{\mathrm{UV}}}+\frac{c(\epsilon)-1}{\epsilon}, \\
& \frac{c(\epsilon)}{\epsilon_{\mathrm{IR}}}=\frac{1}{\epsilon_{\mathrm{IR}}}+\frac{c(\epsilon)-1}{\epsilon},
\end{aligned}
$$

where we have dropped the subscripts "UV" and "IR" in the second terms of the above equations because those terms are finite. Hence, we have

$$
c(\epsilon)\left(\frac{1}{2 \epsilon_{\mathrm{UV}}}-\frac{1}{2 \epsilon_{\mathrm{IR}}}\right)=\frac{1}{2 \epsilon_{\mathrm{UV}}}-\frac{1}{2 \epsilon_{\mathrm{IR}}} .
$$

Therefore,

$$
\left\langle 0\left|\mathcal{O}_{0}^{\left.Q \bar{Q}^{(3} S_{1}^{[1]}\right)}\left({ }^{3} P^{[8]}\right)\right| 0\right\rangle^{(1)}=\frac{8 \alpha_{s}}{3 \pi m^{2}}\left(\frac{1}{2 \epsilon_{\mathrm{UV}}}-\frac{1}{2 \epsilon_{\mathrm{IR}}}\right) \frac{N_{c}^{2}-1}{4 N_{c}^{2}}\left(\xi^{\dagger} q^{i} q^{\prime l} \sigma^{k} \eta \eta^{\dagger} q^{i} q^{l} \sigma^{k} \xi\right)_{{ }^{3} S_{1}^{[1]}} .
$$

\footnotetext{
${ }^{5}$ This approach was first used in Appendix B of Ref. [2]. It has been discussed subsequently in Refs. [29, 41, 42]. In Ref. [43], it was pointed out that this approach allocates contributions that are infrared finite to the short-distance coefficients, rather than to the LDMEs, and is, therefore, the NRQCD analogue of the standard methods for computing dimensionally regulated short-distance coefficients for hard-scattering processes in collinear factorization in QCD.
} 
We renormalize $\left\langle 0\left|\mathcal{O}_{0}^{Q \bar{Q}\left({ }^{3} S_{1}^{[1]}\right)}\left({ }^{3} P^{[8]}\right)\right| 0\right\rangle^{(1)}$ in the $\overline{\mathrm{MS}}$ scheme. In the $\overline{\mathrm{MS}}$ scheme, one constructs the counterterm for a UV-divergent subdiagram by subtracting the poles in $\epsilon_{\mathrm{UV}}$ that appear in that subdiagram. ${ }^{6}$ In order to insure that the counterterm contribution to an LDME removes precisely the contribution that is proportional to the UV divergence in the divergent subdiagram, one must compute all factors that are external to the divergent subdiagram, such as the projections of external momenta onto particular angular-momentum states, in $d=4-2 \epsilon$ dimensions. Hence, we find that the $\overline{\mathrm{MS}}$-counterterm contribution to $\left\langle 0\left|\mathcal{O}_{0}^{Q \bar{Q}\left({ }^{3} S_{1}^{[1]}\right)}\left({ }^{3} P^{[8]}\right)\right| 0\right\rangle^{(1)}$ is

$$
\begin{aligned}
\delta\left\langle 0\left|\mathcal{O}_{0}^{Q \bar{Q}\left({ }^{3} S_{1}^{[1]}\right)}\left({ }^{3} P^{[8]}\right)\right| 0\right\rangle^{(1)} & =\frac{8 \alpha_{s}}{3 \pi m^{2}}\left(\frac{-1}{2 \epsilon_{\mathrm{UV}}}\right) \frac{N_{c}^{2}-1}{4 N_{c}^{2}}\left(\xi^{\dagger} q^{\prime i} q^{\prime l} \sigma^{k} \eta \eta^{\dagger} q^{i} q^{l} \sigma^{k} \xi\right)_{{ }^{3} S_{1}^{[1]}} \\
& =\left[Z^{(1)}\left({ }^{3} P^{[8]} \rightarrow{ }^{3} S_{1}^{[1]}\right)-1\right]\left(\xi^{\dagger} q^{\prime i} q^{\prime l} \sigma^{k} \eta \eta^{\dagger} q^{i} q^{l} \sigma^{k} \xi\right)_{{ }^{3} S_{1}^{[1]}} .
\end{aligned}
$$

Then, we have

$$
\begin{aligned}
\left\langle 0\left|\mathcal{O}_{0}^{Q \bar{Q}\left({ }^{3} S_{1}^{[1]}\right)}\left({ }^{3} P^{[8]}\right)\right| 0\right\rangle \frac{(1)}{\mathrm{MS}} & =\left\langle 0\left|\mathcal{O}_{0}^{Q \bar{Q}\left({ }^{3} S_{1}^{[1]}\right)}\left({ }^{3} P^{[8]}\right)\right| 0\right\rangle^{(1)}+\delta\left\langle 0\left|\mathcal{O}_{0}^{Q \bar{Q}\left({ }^{3} S_{1}^{[1]}\right)}\left({ }^{3} P^{[8]}\right)\right| 0\right\rangle^{(1)} \\
& =\frac{8 \alpha_{s}}{3 \pi m^{2}}\left(\frac{-1}{2 \epsilon_{\mathrm{IR}}}\right) \frac{N_{c}^{2}-1}{4 N_{c}^{2}}\left(\xi^{\dagger} q^{i} q^{\prime l} \sigma^{k} \eta \eta^{\dagger} q^{i} q^{l} \sigma^{k} \xi\right)_{{ }_{3} S_{1}^{[1]}}
\end{aligned}
$$

Now we extract the $S$-wave part by averaging over the angles of $\boldsymbol{q}$ and $\boldsymbol{q}^{\prime}$ :

$$
\left\langle 0\left|\mathcal{O}_{0}^{\left.Q \bar{Q}^{3} S_{1}^{[1]}\right)}\left({ }^{3} P^{[8]}\right)\right| 0\right\rangle \frac{(1)}{\mathrm{MS}}=\frac{8 \alpha_{s}}{3 \pi m^{2}}\left(\frac{-1}{2 \epsilon_{\mathrm{IR}}}\right) \frac{N_{c}^{2}-1}{4 N_{c}^{2}} \frac{1}{d-1}\left\langle 0\left|\mathcal{O}_{4,1}^{\left.Q \bar{Q}^{(3} S_{1}^{[1]}\right)}\left({ }^{3} S_{1}^{[1]}\right)\right| 0\right\rangle^{(0)} .
$$

\subsection{2 ${ }^{3} S_{1}^{[8]} \rightarrow{ }^{3} P^{[8]}$}

In order $\alpha_{s}$, the ${ }^{3} S_{1}^{[8]} Q \bar{Q}$ operator can couple to the $Q \bar{Q}\left({ }^{3} P^{[8]}\right)$ state. By carrying out a calculation that is very similar to the one in the preceding section, we find that

$$
Z^{(1)}\left({ }^{3} S_{1}^{[8]} \rightarrow{ }^{3} P^{[8]}\right)-1=\frac{8 \alpha_{s}}{3 \pi m^{2}}\left(\frac{-1}{2 \epsilon_{\mathrm{UV}}}\right) \frac{N_{c}^{2}-4}{4 N_{c}}
$$

and that

$$
\left\langle 0\left|\mathcal{O}_{0}^{Q \bar{Q}\left({ }^{3} P^{[8]}\right)}\left({ }^{3} S_{1}^{[8]}\right)\right| 0\right\rangle_{\mathrm{MS}}^{(1)}=\frac{8 \alpha_{s}}{3 \pi m^{2}}\left(\frac{-1}{2 \epsilon_{\mathrm{IR}}}\right) \frac{N_{c}^{2}-4}{4 N_{c}}\left\langle 0\left|\mathcal{O}_{0}^{Q \bar{Q}\left({ }^{3} P^{[8]}\right)}\left({ }^{3} P^{[8]}\right)\right| 0\right\rangle^{(0)} .
$$

The equivalent result for NRQCD decay LDMEs was obtained in Ref. [40].

\subsection{Order $\alpha_{s}^{2}$}

In order $\alpha_{s}^{2}$, the ${ }^{3} S_{1}^{[8]} Q \bar{Q}$ operator can couple to the $Q \bar{Q}\left({ }^{3} S_{1}^{[1]}\right)$ state through the diagrams that are shown in Fig. 6. As in the order- $\alpha_{s}$ case, we expand the integrands for these

\footnotetext{
${ }^{6}$ In some versions of the $\overline{\mathrm{MS}}$ scheme, one subtracts constants, as well as poles, in constructing the counterterms. These constants are accounted for in method that we use in this paper by the factors $\left(\mu^{2} e^{\gamma_{\mathrm{E}}}\right)^{2 \epsilon}$ that are associated with $g_{s}^{2}$.
} 


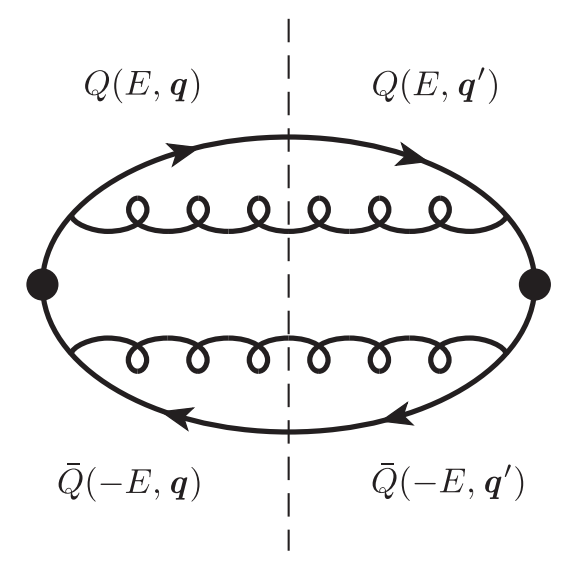

Figure 6. One of the 36 Feynman diagrams for computing the LDME $\left\langle 0\left|\mathcal{O}_{n}^{Q \bar{Q}\left({ }^{3} S_{1}^{[1]}\right)}\left[Q \bar{Q}\left({ }^{3} S_{1}^{[8]}\right)\right]\right| 0\right\rangle^{(2)}$. The solid circles represent the $Q \bar{Q}$ operators in the LDME. Thirtyfive additional diagrams can be obtained by permuting the gluon-fermion and operator vertices on the left and right sides of the cut.

diagrams in powers of $1 / m$ and, again, only the leading power contributes in dimensional regularization. Now we combine contributions that differ in the order of the gluon vertices on a quark or an antiquark line by making use of the fact that the color factor for the color-singlet contribution is symmetric under the interchange of the gluon color indices and by making use of the identity

$$
\frac{1}{|\boldsymbol{k}|} \frac{1}{|\boldsymbol{k}|+|\boldsymbol{\ell}|}+\frac{1}{|\boldsymbol{\ell}|} \frac{1}{|\boldsymbol{k}|+|\boldsymbol{\ell}|}=\frac{1}{|\boldsymbol{k}|} \frac{1}{|\boldsymbol{\ell}|} .
$$

After we combine the contributions of all of the diagrams in this way, the loop integrations decouple, and we have

$$
\begin{aligned}
\left\langle 0\left|\mathcal{O}_{0}^{\left.Q \bar{Q}{ }^{3} S_{1}^{[1]}\right)}\left({ }^{3} S_{1}^{[8]}\right)\right| 0\right\rangle^{(2)}= & \frac{\left(4 \pi \alpha_{s}\right)^{2}}{m^{4}}\left(\frac{\mu^{2}}{4 \pi} e^{\gamma_{\mathrm{E}}}\right)^{2 \epsilon} \frac{\left(N_{c}^{2}-4\right)\left(N_{c}^{2}-1\right)}{8 N_{c}^{3}} \\
& \times\left(\xi^{\dagger} q^{\prime i} q^{\prime r} \sigma^{n} \eta \eta^{\dagger} q^{j} q^{s} \sigma^{n} \xi\right)_{{ }^{3} S_{1}^{[1]}} \\
& \times \int \frac{d^{d-1} k}{(2 \pi)^{d-1}} \frac{\delta^{i j}-\hat{\boldsymbol{k}}^{i} \hat{\boldsymbol{k}}^{j}}{|\boldsymbol{k}|^{3}} \int \frac{d^{d-1} \ell}{(2 \pi)^{d-1}} \frac{\delta^{r s}-\hat{\boldsymbol{\ell}}^{r} \hat{\boldsymbol{\ell}}^{s}}{|\boldsymbol{\ell}|^{3}} \\
= & \frac{1}{2}\left[\frac{8 \alpha_{s}}{3 \pi m^{2}}\left(\frac{1}{2 \epsilon_{\mathrm{UV}}}-\frac{1}{2 \epsilon_{\mathrm{IR}}}\right)\right]^{2} \frac{\left(N_{c}^{2}-1\right)\left(N_{c}^{2}-4\right)}{16 N_{c}^{3}} \\
& \times\left(\xi^{\dagger} q^{\prime i} q^{\prime r} \sigma^{n} \eta \eta^{\dagger} q^{i} q^{r} \sigma^{n} \xi\right)_{{ }^{3} S_{1}^{[1]}},
\end{aligned}
$$

where we have made use of Eq. (6.9).

We carry out the renormalization of $\left\langle 0\left|\mathcal{O}_{0}^{\left.Q \bar{Q}^{(3} S_{1}^{[1]}\right)}\left({ }^{3} S_{1}^{[8]}\right)\right| 0\right\rangle^{(2)}$ in the $\overline{\mathrm{MS}}$ scheme. First, we add the contribution of the one-loop diagrams involving the counterterm $Z^{(1)}\left({ }^{3} P^{[8]} \rightarrow\right.$ $\left.{ }^{3} S_{1}^{[1]}\right)-1$, which are shown in Fig. 7. The contribution of these diagrams is

$$
\delta_{1}\left\langle 0\left|\mathcal{O}_{0}^{Q \bar{Q}\left({ }^{3} S_{1}^{[1]}\right)}\left({ }^{3} S_{1}^{[8]}\right)\right| 0\right\rangle^{(2)}=\left[Z^{(1)}\left({ }^{3} S_{1}^{[8]} \rightarrow{ }^{3} P^{[8]}\right)-1\right]\left\langle 0\left|\mathcal{O}_{0}^{Q \bar{Q}\left({ }^{3} S_{1}^{[1]}\right)}\left({ }^{3} P^{[8]}\right)\right| 0\right\rangle^{(1)},
$$




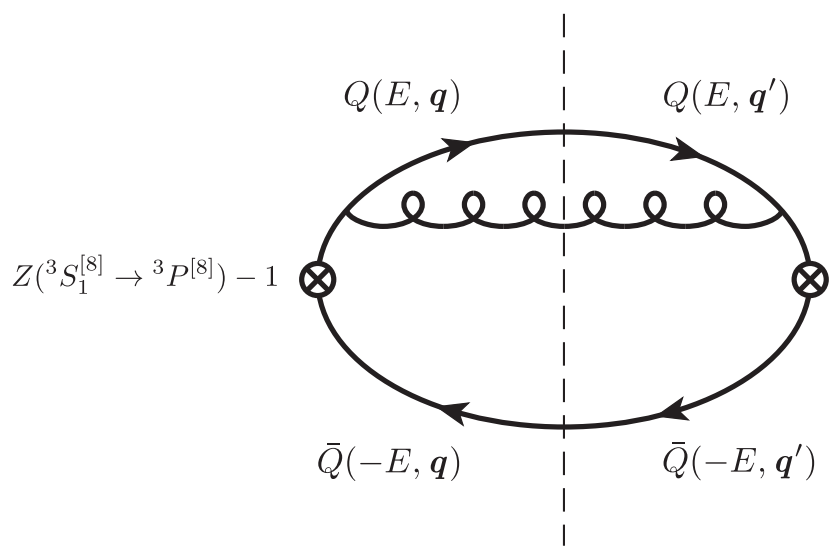

Figure 7. One of the four Feynman diagrams for computing the one-loop correction to the counterterm $Z\left({ }^{3} S_{1}^{[8]} \rightarrow{ }^{3} P^{[8]}\right)-1$. The symbols $\otimes$ represent the counterterm $Z\left({ }^{3} S_{1}^{[8]} \rightarrow{ }^{3} P^{[8]}\right)-1$. Three additional diagrams can be obtained by permuting the gluon-fermion and counterterm vertices on the left and right sides of the cut.

where $\left\langle 0\left|\mathcal{O}_{0}^{Q \bar{Q}\left({ }^{3} S_{1}^{[1]}\right)}\left({ }^{3} P^{[8]}\right)\right| 0\right\rangle^{(1)}$ is given in Eq. (6.10) and $Z^{(1)}\left({ }^{3} S_{1}^{[8]} \rightarrow{ }^{3} P^{[8]}\right)-1$ is given in Eq. (6.14). Thus,

$$
\begin{aligned}
\delta_{1}\left\langle 0\left|\mathcal{O}_{0}^{Q \bar{Q}\left({ }^{3} S_{1}^{[1]}\right)}\left({ }^{3} S_{1}^{[8]}\right)\right| 0\right\rangle^{(2)}= & -\frac{8 \alpha_{s}}{3 \pi m^{2}}\left(\frac{1}{2 \epsilon_{\mathrm{UV}}}-\frac{1}{2 \epsilon_{\mathrm{IR}}}\right) \frac{\left(N_{c}^{2}-1\right)\left(N_{c}^{2}-4\right)}{16 N_{c}^{3}} \\
& \times \frac{8 \alpha_{s}}{3 \pi m^{2}} \frac{1}{2 \epsilon_{\mathrm{UV}}}\left(\xi^{\dagger} q^{\prime i} q^{\prime r} \sigma^{n} \eta \eta^{\dagger} q^{i} q^{r} \sigma^{n} \xi\right)_{{ }^{3} S_{1}^{[1]}} .
\end{aligned}
$$

Adding this counterterm contribution to Eq. (6.17), we obtain

$$
\begin{aligned}
& \left\langle 0\left|\mathcal{O}_{0}^{Q \bar{Q}\left({ }^{3} S_{1}^{[1]}\right)}\left({ }^{3} S_{1}^{[8]}\right)\right| 0\right\rangle^{(2)}+\delta_{1}\left\langle 0\left|\mathcal{O}_{0}^{Q \bar{Q}\left({ }^{3} S_{1}^{[1]}\right)}\left({ }^{3} S_{1}^{[8]}\right)\right| 0\right\rangle^{(2)} \\
& =\frac{1}{8}\left(\frac{8 \alpha_{s}}{3 \pi m^{2}}\right)^{2}\left(\frac{-1}{\epsilon_{\mathrm{UV}}^{2}}+\frac{1}{\epsilon_{\mathrm{IR}}^{2}}\right) \frac{\left(N_{c}^{2}-1\right)\left(N_{c}^{2}-4\right)}{16 N_{c}^{3}}\left(\xi^{\dagger} q^{\prime i} q^{\prime r} \sigma^{n} \eta \eta^{\dagger} q^{i} q^{r} \sigma^{n} \xi\right)_{{ }^{3} S_{1}^{[1]}}
\end{aligned}
$$

We see that the counterterm contribution removes the cross term between the pole in $\epsilon_{\mathrm{UV}}$ and the pole in $\epsilon_{\mathrm{IR}}$. Hence, the remaining overall UV divergence is not coupled to infrared contributions, as is required for the consistency of the renormalization program. There are no single poles in $\epsilon_{\mathrm{UV}}$. The remaining double pole in $\epsilon_{\mathrm{UV}}$ is removed by adding the $\overline{\mathrm{MS}}$-counterterm contribution

$$
\begin{aligned}
\delta_{2}\left\langle 0\left|\mathcal{O}_{0}^{Q \bar{Q}\left({ }^{3} S_{1}^{[1]}\right)}\left({ }^{3} S_{1}^{[8]}\right)\right| 0\right\rangle^{(2)}= & \left(\frac{8 \alpha_{s}}{3 \pi m^{2}}\right)^{2} \frac{1}{8 \epsilon_{\mathrm{UV}}^{2}} \frac{\left(N_{c}^{2}-1\right)\left(N_{c}^{2}-4\right)}{16 N_{c}^{3}} \\
& \times\left(\xi^{\dagger} q^{i} q^{\prime r} \sigma^{n} \eta \eta^{\dagger} q^{i} q^{r} \sigma^{n} \xi\right)^{{ }^{3} S_{1}^{[1]}} \\
= & {\left[Z^{(2)}\left({ }^{3} S_{1}^{[8]} \rightarrow{ }^{3} S_{1}^{[1]}\right)-1\right]\left(\xi^{\dagger} q^{\prime i} q^{\prime r} \sigma^{n} \eta \eta^{\dagger} q^{i} q^{r} \sigma^{n} \xi\right)_{{ }_{3} S_{1}^{[1]}} . }
\end{aligned}
$$


Adding this counterterm contribution to Eq. (6.20), we obtain

$$
\begin{aligned}
\left\langle 0\left|\mathcal{O}_{0}^{Q \bar{Q}\left({ }^{3} S_{1}^{[1]}\right)}\left({ }^{3} S_{1}^{[8]}\right)\right| 0\right\rangle \frac{(2)}{\mathrm{MS}}= & \left\langle 0\left|\mathcal{O}_{0}^{Q \bar{Q}\left({ }^{3} S_{1}^{[1]}\right)}\left({ }^{3} S_{1}^{[8]}\right)\right| 0\right\rangle^{(2)}+\delta_{1}\left\langle 0\left|\mathcal{O}_{0}^{Q \bar{Q}\left({ }^{3} S_{1}^{[1]}\right)}\left({ }^{3} S_{1}^{[8]}\right)\right| 0\right\rangle^{(2)} \\
& +\delta_{2}\left\langle 0\left|\mathcal{O}_{0}^{Q \bar{Q}\left({ }^{3} S_{1}^{[1]}\right)}\left({ }^{3} S_{1}^{[8]}\right)\right| 0\right\rangle^{(2)} \\
= & \left(\frac{8 \alpha_{s}}{3 \pi m^{2}}\right)^{2} \frac{1}{8 \epsilon_{\mathrm{IR}}^{2}} \frac{\left(N_{c}^{2}-1\right)\left(N_{c}^{2}-4\right)}{16 N_{c}^{3}} \\
& \times\left(\xi^{\dagger} q^{i} q^{\prime r} \sigma^{n} \eta \eta^{\dagger} q^{i} q^{r} \sigma^{n} \xi\right)_{{ }_{3} S_{1}^{[1]}}
\end{aligned}
$$

We evaluate the $S$-wave part by carrying out the average over the directions of $\boldsymbol{q}$ and $\boldsymbol{q}^{\prime}$, with the result

$$
\begin{aligned}
\left\langle 0\left|\mathcal{O}_{0}^{Q \bar{Q}\left({ }^{3} S_{1}^{[1]}\right)}\left({ }^{3} S_{1}^{[8]}\right)\right| 0\right\rangle \frac{(2)}{\mathrm{MS}}= & \frac{1}{8 \epsilon_{\mathrm{IR}}^{2}}\left(\frac{8 \alpha_{s}}{3 \pi m^{2}}\right)^{2} \frac{\left(N_{c}^{2}-1\right)\left(N_{c}^{2}-4\right)}{16 N_{c}^{3}} \\
& \times \frac{1}{d-1}\left\langle 0\left|\mathcal{O}_{4,1}^{Q \bar{Q}\left({ }^{3} S_{1}^{[1]}\right)}\left({ }^{3} S_{1}^{[1]}\right)\right| 0\right\rangle^{(0)}
\end{aligned}
$$

\subsection{Renormalization-group evolution}

In the renormalized LDMEs, we can identify the dimensional-regularization scale $\mu$ with the NRQCD factorization scale $\mu_{\Lambda}$. We now work out the renormalization-group evolution with respect to $\mu_{\Lambda}$ of the LDMEs $\left\langle 0\left|\mathcal{O}_{0}^{\left.Q \bar{Q}^{3} S_{1}^{[1]}\right)}\left({ }^{3} P^{[8]}\right)\right| 0\right\rangle \frac{(1)}{\mathrm{MS}},\left\langle 0\left|\mathcal{O}_{0}^{Q \bar{Q}\left({ }^{3} P^{[8]}\right)}\left({ }^{3} S_{1}^{[8]}\right)\right| 0\right\rangle \frac{(1)}{\mathrm{MS}}$, and $\left\langle 0\left|\mathcal{O}_{0}^{Q \bar{Q}\left({ }^{3} S_{1}^{[1]}\right)}\left({ }^{3} S_{1}^{[8]}\right)\right| 0\right\rangle \frac{(2)}{\mathrm{MS}}$. First, we take $d / d \log \mu_{\Lambda}$ of Eq. (6.13):

$$
\begin{aligned}
\frac{d}{d \log \mu_{\Lambda}}\left\langle 0\left|\mathcal{O}_{0}^{\left.Q \bar{Q}^{(3} S_{1}^{[1]}\right)}\left({ }^{3} P^{[8]}\right)\right| 0\right\rangle \frac{(1)}{\mathrm{MS}} & =\frac{d \alpha_{s}}{d \log \mu_{\Lambda}} \frac{d}{d \alpha_{s}}\left\langle 0\left|\mathcal{O}_{0}^{Q \bar{Q}\left({ }^{3} S_{1}^{[1]}\right)}\left({ }^{3} P^{[8]}\right)\right| 0\right\rangle \frac{(1)}{\mathrm{MS}} \\
& =\frac{8 \alpha_{s}}{3 \pi m^{2}} \frac{N_{c}^{2}-1}{4 N_{c}^{2}} \frac{1}{d-1}\left\langle 0\left|\mathcal{O}_{4,1}^{Q \bar{Q}\left({ }^{3} S_{1}^{[1]}\right)}\left({ }^{3} S_{1}^{[1]}\right)\right| 0\right\rangle^{(0)}
\end{aligned}
$$

where we have used

$$
\frac{d \alpha_{s}}{d \log \mu_{\Lambda}}=-2 \epsilon \alpha_{s}+O\left(\alpha_{s}^{2}\right) .
$$

The result in Eq. (6.24) agrees with the corresponding results of Refs. [23, 44].

Similarly, taking $d / d \log \mu_{\Lambda}$ of Eq. (6.15), we obtain

$$
\frac{d}{d \log \mu_{\Lambda}}\left\langle 0\left|\mathcal{O}_{0}^{\left.Q \bar{Q}^{(3} P^{[8]}\right)}\left({ }^{3} S_{1}^{[8]}\right)\right| 0\right\rangle \frac{(1)}{\mathrm{MS}}=\frac{8 \alpha_{s}}{3 \pi m^{2}} \frac{N_{c}^{2}-4}{4 N_{c}}\left\langle 0\left|\mathcal{O}_{0}^{Q \bar{Q}\left({ }^{3} P^{[8]}\right)}\left({ }^{3} P^{[8]}\right)\right| 0\right\rangle^{(0)} .
$$

Taking $d / d \log \mu_{\Lambda}$ of Eq. (6.23), we also obtain

$$
\begin{aligned}
\frac{d}{d \log \mu_{\Lambda}}\left\langle 0\left|\mathcal{O}_{0}^{Q \bar{Q}\left({ }^{3} S_{1}^{[1]}\right)}\left({ }^{3} S_{1}^{[8]}\right)\right| 0\right\rangle \frac{(2)}{\mathrm{MS}}= & \left(\frac{8 \alpha_{s}}{3 \pi m^{2}}\right)^{2}\left(\frac{-1}{2 \epsilon_{\mathrm{IR}}}\right) \frac{\left(N_{c}^{2}-1\right)\left(N_{c}^{2}-4\right)}{16 N_{c}^{3}} \\
& \times \frac{1}{d-1}\left\langle 0\left|\mathcal{O}_{4,1}^{Q \bar{Q}\left({ }^{3} S_{1}^{[1]}\right)}\left({ }^{3} S_{1}^{[1]}\right)\right| 0\right\rangle^{(0)}
\end{aligned}
$$


Substituting Eq. (6.13) into the right side of Eq. (6.27) we find that

$$
\frac{d}{d \log \mu_{\Lambda}}\left\langle 0\left|\mathcal{O}_{0}^{Q \bar{Q}\left({ }^{3} S_{1}^{[1]}\right)}\left({ }^{3} S_{1}^{[8]}\right)\right| 0\right\rangle \frac{(2)}{\mathrm{MS}}=\frac{8 \alpha_{s}}{3 \pi m^{2}} \frac{N_{c}^{2}-4}{4 N_{c}}\left\langle 0\left|\mathcal{O}_{0}^{Q \bar{Q}\left({ }^{3} S_{1}^{[1]}\right)}\left({ }^{3} P^{[8]}\right)\right| 0\right\rangle_{\overline{\mathrm{MS}}}^{(1)}
$$

Equations (6.26) and (6.28) agree with the result in Eq. (B19b) of Ref. [2] at the leading nontrivial order in $v$ and with the corresponding result in Ref [44], but disagree with the corresponding result in Ref. [23].

\section{NRQCD matching and short-distance coefficients}

In the discussion to follow, and in remainder of this paper, the short-distance coefficients $d_{n}$ that appear are always in the $\overline{\mathrm{MS}}$ scheme. For brevity, we do not indicate the scheme explicitly.

In relative order $v^{4}$, the NRQCD factorization equation that relates full QCD and NRQCD for the fragmentation of a gluon into a ${ }^{3} S_{1}^{[1]} Q \bar{Q}$ pair is

$$
\begin{aligned}
D_{4}\left[g \rightarrow Q \bar{Q}\left({ }^{3} S_{1}^{[1]}\right)\right]^{(3)}= & \left.\left\{d_{4,1}\left[g \rightarrow Q \bar{Q}\left({ }^{3} S_{1}^{[1]}\right)\right]\right]^{(3)}+d_{4,2}\left[g \rightarrow Q \bar{Q}\left({ }^{3} S_{1}^{[1]}\right)\right]^{(3)}\right\} \\
& \times\left\langle 0\left|\mathcal{O}_{4}^{Q \bar{Q}\left({ }^{3} S_{1}^{[1]}\right)}\left({ }^{3} S_{1}^{[1]}\right)\right| 0\right\rangle^{(0)} \\
+ & d_{0}\left[g \rightarrow Q \bar{Q}\left({ }^{3} P^{[8]}\right)\right]^{(2)}\left\langle 0\left|\mathcal{O}_{0}^{Q \bar{Q}\left({ }^{3} S_{1}^{[1]}\right)}\left({ }^{3} P^{[8]}\right)\right| 0\right\rangle^{(1)} \\
+ & d_{0}\left[g \rightarrow Q \bar{Q}\left({ }^{3} S_{1}^{[8]}\right)\right]^{(1)}\left\langle 0\left|\mathcal{O}_{0}^{Q \bar{Q}\left({ }^{3} S_{1}^{[1]}\right)}\left({ }^{3} S_{1}^{[8]}\right)\right| 0\right\rangle^{(2)}
\end{aligned}
$$

where we have shown the contributions at the leading nontrivial order in $\alpha_{s}$, namely, $\alpha_{s}^{3}$, and the LDME $\left\langle 0\left|\mathcal{O}_{4}^{Q \bar{Q}\left({ }^{3} S_{1}^{[1]}\right)}\left({ }^{3} S_{1}^{[1]}\right)\right| 0\right\rangle^{(0)}$ means either $\left\langle 0\left|\mathcal{O}_{4,1}^{Q \bar{Q}\left({ }^{3} S_{1}^{[1]}\right)}\left({ }^{3} S_{1}^{[1]}\right)\right| 0\right\rangle^{(0)}$ or $\langle 0| \mathcal{O}_{4,2}^{Q \bar{Q}\left({ }^{3} S_{1}^{[1]}\right)}$ $\left({ }^{3} S_{1}^{[1]}\right)|0\rangle^{(0)}$, since they are equal at the present order of interest in $v$, as we have explained in Sec. 3. We wish to use this matching equation to determine the sum of short-distance coefficients $d_{4,1}\left[g \rightarrow Q \bar{Q}\left({ }^{3} S_{1}^{[1]}\right)\right]^{(3)}+d_{4,2}\left[g \rightarrow Q \bar{Q}\left({ }^{3} S_{1}^{[1]}\right)\right]^{(3)}$. We have already computed the $Q \bar{Q}$ LDMEs on the right side of Eq. (7.1). In order to fix the short-distance coefficients in the second and third terms on the right side of Eq. (7.1), we make use of a matching equation at relative order $v^{2}$, which is

$$
\begin{aligned}
D_{2}\left[g \rightarrow Q \bar{Q}\left({ }^{3} P^{[8]}\right)\right]^{(2)} & =d_{0}\left[g \rightarrow Q \bar{Q}\left({ }^{3} P^{[8]}\right)\right]^{(2)}\left\langle 0\left|\mathcal{O}_{0}^{Q \bar{Q}\left({ }^{3} P^{[8]}\right)}\left({ }^{3} P^{[8]}\right)\right| 0\right\rangle^{(0)} \\
& +d_{0}\left[g \rightarrow Q \bar{Q}\left({ }^{3} S_{1}^{[8]}\right)\right]^{(1)}\left\langle 0\left|\mathcal{O}_{0}^{Q \bar{Q}\left({ }^{3} P^{[8]}\right)}\left({ }^{3} S_{1}^{[8]}\right)\right| 0\right\rangle^{(1)}
\end{aligned}
$$

and a matching equation at relative order $v^{0}$, which is

$$
D_{0}\left[g \rightarrow Q \bar{Q}\left({ }^{3} S_{1}^{[8]}\right)\right]^{(1)}=d_{0}\left[g \rightarrow Q \bar{Q}\left({ }^{3} S_{1}^{[8]}\right)\right]^{(1)}\left\langle 0\left|\mathcal{O}_{0}^{Q \bar{Q}\left({ }^{3} S_{1}^{[8]}\right)}\left({ }^{3} S_{1}^{[8]}\right)\right| 0\right\rangle^{(0)} .
$$

First, we solve Eq. (7.3) for $d_{0}\left[g \rightarrow Q \bar{Q}\left({ }^{3} S_{1}^{[8]}\right)\right]^{(1)}$, making use of Eq. (5.13) for $D_{0}[g \rightarrow$ $\left.Q \bar{Q}\left({ }^{3} S_{1}^{[8]}\right)\right]^{(1)}$ and Eq. (6.1c) for $\left\langle 0\left|\mathcal{O}_{0}^{Q \bar{Q}\left({ }^{3} S_{1}^{[8]}\right)}\left({ }^{3} S_{1}^{[8]}\right)\right| 0\right\rangle^{(0)}$. The result is

$$
d_{0}\left[g \rightarrow Q \bar{Q}\left({ }^{3} S_{1}^{[8]}\right)\right]^{(1)}=\frac{\pi \alpha_{s}}{(d-1)\left(N_{c}^{2}-1\right) m^{3}}\left(\frac{\mu_{\Lambda}^{2}}{4 \pi} e^{\gamma_{\mathrm{E}}}\right)^{\epsilon} \delta(1-z) .
$$


The short-distance coefficient in Eq. (7.4) agrees with that in Refs. [30, 31].

Next, we determine the short-distance coefficient $d_{0}\left[g \rightarrow Q \bar{Q}\left({ }^{3} P^{[8]}\right)\right]^{(2)}$ by making use of the order- $v^{2}$ matching equation (7.2). We substitute $D_{2}\left[g \rightarrow Q \bar{Q}\left({ }^{3} P^{[8]}\right)\right]^{(2)}$ in Eq. (5.16), $d_{0}\left[g \rightarrow Q \bar{Q}\left({ }^{3} S_{1}^{[8]}\right)\right]^{(1)}$ in Eq. (7.4), $\left\langle 0\left|\mathcal{O}_{0}^{Q \bar{Q}\left({ }^{3} P^{[8]}\right)}\left({ }^{3} P^{[8]}\right)\right| 0\right\rangle^{(0)}$ in Eq. (6.1f), and $\left\langle 0\left|\mathcal{O}_{0}^{Q \bar{Q}\left({ }^{3} P^{[8]}\right)}\left({ }^{3} S_{1}^{[8]}\right)\right| 0\right\rangle^{(1)}$ in Eq. (6.15) into Eq. (7.2). Then, solving for $d_{0}\left[g \rightarrow Q \bar{Q}\left({ }^{3} P^{[8]}\right)\right]^{(2)}$, we obtain

$$
\begin{aligned}
d_{0}\left[g \rightarrow Q \bar{Q}\left({ }^{3} P^{[8]}\right)\right]^{(2)}= & \frac{8 \alpha_{s}^{2}}{3(d-1)\left(N_{c}^{2}-1\right) m^{5}}\left(\frac{N_{c}^{2}-4}{4 N_{c}}\right)\left(\frac{\mu_{\Lambda}^{2}}{4 \pi} e^{\gamma_{\mathrm{E}}}\right)^{\epsilon} \\
& \times\left\{-\frac{\delta(1-z)}{2 \epsilon_{\mathrm{IR}}}\left[\frac{(1-\epsilon) \Gamma(1+\epsilon)}{1-\frac{2}{3} \epsilon}\left(\frac{\mu_{\Lambda}^{2}}{4 m^{2}} e^{\gamma_{\mathrm{E}}}\right)^{\epsilon}-1\right]\right. \\
& \left.+\frac{(1-\epsilon) \Gamma(1+\epsilon)}{1-\frac{2}{3} \epsilon}\left(\frac{\mu_{\Lambda}^{2}}{4 m^{2}} e^{\gamma_{\mathrm{E}}}\right)^{\epsilon} f(z)\right\}
\end{aligned}
$$

where $f(z)$ is given in Eq. (5.16b). In Ref. [29], the color-singlet short-distance coefficients $d_{0}\left[g \rightarrow Q \bar{Q}\left({ }^{3} P_{J}^{[1]}\right)\right]^{(2)}$ were computed. Summing the results for $d_{0}\left[g \rightarrow Q \bar{Q}\left({ }^{3} P_{J}^{[1]}\right)\right]^{(2)}$ in Ref. [29] over $J=0,1$ and 2 and multiplying by $\left[\left(N_{c}^{2}-1\right) /\left(4 N_{c}^{2}\right)\right]^{-1}\left[\left(N_{c}^{2}-4\right) /\left(4 N_{c}\right)\right]$ in order to obtain the corresponding short-distance coefficient for the color-octet channel, we find agreement with our result in Eq. (7.5).

The expression in Eq. (7.5) gives the exact $\epsilon$ dependence. Expanding this expression to order $\epsilon^{1}$, using the expression for $f(z)$ in Eq. (5.20), we find that

$$
\begin{aligned}
d_{0}[g \rightarrow & \left.Q \bar{Q}\left({ }^{3} P^{[8]}\right)\right]^{(2)} \\
= & \frac{8 \alpha_{s}^{2}}{3(d-1)\left(N_{c}^{2}-1\right) m^{5}} \frac{N_{c}^{2}-4}{4 N_{c}}\left(\frac{\mu_{\Lambda}^{2}}{4 \pi} e^{\gamma_{\mathrm{E}}}\right)^{\epsilon} \\
\times & \left\{\frac{1}{6} \delta(1-z)\left[1-6 \log \frac{\mu_{\Lambda}}{2 m}+\epsilon\left(\frac{2}{3}-\frac{\pi^{2}}{4}+2 \log \frac{\mu_{\Lambda}}{2 m}-6 \log ^{2} \frac{\mu_{\Lambda}}{2 m}\right)\right]\right. \\
& +\left(\frac{1}{1-z}\right)_{+}\left[1-\epsilon\left(\frac{1}{3}-2 \log \frac{\mu_{\Lambda}}{2 m}\right)\right]-2 \epsilon\left[\frac{\log (1-z)}{1-z}\right]_{+} \\
& +\frac{13-7 z}{4}\left[1-\epsilon\left(\frac{1}{3}+\frac{3}{2} \log (1-z)-2 \log \frac{\mu_{\Lambda}}{2 m}\right)\right] \log (1-z) \\
& -\frac{1}{8}(1-2 z)(8-5 z)\left(1+2 \epsilon \log \frac{\mu_{\Lambda}}{2 m}\right) \\
& \left.+\frac{\epsilon}{24}\left[8+48 z-38 z^{2}+15\left(8-11 z+4 z^{2}\right) \log (1-z)\right]\right\}+O\left(\epsilon^{2}\right) .
\end{aligned}
$$

Finally, we determine the sum of short-distance coefficients $d_{4,1}\left[g \rightarrow Q \bar{Q}\left({ }^{3} S_{1}^{[1]}\right)\right]^{(3)}+$ $d_{4,2}\left[g \rightarrow Q \bar{Q}\left({ }^{3} S_{1}^{[1]}\right)\right]^{(3)}$ by making use of the matching equation at relative order $v^{4}$ [Eq. (7.1)]. We substitute $D_{4}\left[g \rightarrow Q \bar{Q}\left({ }^{3} S_{1}^{[1]}\right)\right]^{(3)}$ in Eq. (5.33), $d_{0}\left[g \rightarrow Q \bar{Q}\left({ }^{3} P^{[8]}\right)\right]^{(2)}$ in Eq. (7.5), $d_{0}[g \rightarrow$ $\left.Q \bar{Q}\left({ }^{3} S_{1}^{[8]}\right)\right]^{(1)}$ in Eq. (7.4), $\left\langle 0\left|\mathcal{O}_{4}^{Q \bar{Q}\left({ }^{3} S_{1}^{[1]}\right)}\left({ }^{3} S_{1}^{[1]}\right)\right| 0\right\rangle^{(0)}$ in Eq. (6.1e), $\left\langle 0\left|\mathcal{O}_{0}^{Q \bar{Q}\left({ }^{3} S_{1}^{[1]}\right)}\left({ }^{3} P^{[8]}\right)\right| 0\right\rangle^{(1)}$ in Eq. (6.13), and $\left\langle 0\left|\mathcal{O}_{0}^{Q \bar{Q}\left({ }^{3} S_{1}^{[1]}\right)}\left({ }^{3} S_{1}^{[8]}\right)\right| 0\right\rangle^{(2)}$ in Eq. (6.23) into Eq. (7.1). Solving for $d_{4,1}[g \rightarrow$ 


$$
\begin{aligned}
\left.Q \bar{Q}\left({ }^{3} S_{1}^{[1]}\right)\right]{ }^{(3)} & +d_{4,2}\left[g \rightarrow Q \bar{Q}\left({ }^{3} S_{1}^{[1]}\right)\right]{ }^{(3)}, \text { we obtain } \\
d_{4,1}[g \rightarrow & \left.\rightarrow \bar{Q}\left({ }^{3} S_{1}^{[1]}\right)\right]^{(3)}+d_{4,2}\left[g \rightarrow Q \bar{Q}\left({ }^{3} S_{1}^{[1]}\right)\right]{ }^{(3)} \\
= & d_{4}\left[g \rightarrow Q \bar{Q}\left({ }^{3} S_{1}^{[1]}\right)\right]^{\text {finite }}+\frac{2 \alpha_{s}^{3}\left(N_{c}^{2}-4\right)}{3 \pi(d-1)^{3} N_{c}^{3} m^{7}}\left\{\delta ( 1 - z ) \left(\frac{1}{24}-\frac{\pi^{2}}{6}\right.\right. \\
& \left.-\frac{1}{3} \log \frac{\mu_{\Lambda}}{2 m}+\log ^{2} \frac{\mu_{\Lambda}}{2 m}\right)+\left(\frac{1}{1-z}\right)_{+}\left(\frac{1}{3}-2 \log \frac{\mu_{\Lambda}}{2 m}\right)+2\left[\frac{\log (1-z)}{1-z}\right]_{+} \\
& -\frac{104-29 z-10 z^{2}}{24}+\frac{7[z+(1+z) \log (1-z)]}{2 z^{2}}+\frac{(1-2 z)(8-5 z)}{4} \log \frac{\mu_{\Lambda}}{2 m} \\
& +\frac{1+z}{4}\left(31-6 z-\frac{36}{z}\right) \log (1-z)-\frac{z}{4}\left(39-6 z+\frac{8}{1-z}\right) \log z \\
& \left.+\frac{13-7 z}{2}\left[\left(\log \frac{1-z}{z^{2}}-\log \frac{\mu_{\Lambda}}{2 m}\right) \log (1-z)-\operatorname{Li}_{2}(z)\right]\right\}+O(\epsilon)
\end{aligned}
$$

where we have used the identity $\operatorname{Li}_{2}(1-z)=\pi^{2} / 6-\operatorname{Li}_{2}(z)-\log z \log (1-z) . d_{4}[g \rightarrow$ $\left.Q \bar{Q}\left({ }^{3} S_{1}^{[1]}\right)\right]^{\text {finite }}$ is defined by

$$
d_{4}\left[g \rightarrow Q \bar{Q}\left({ }^{3} S_{1}^{[1]}\right)\right]^{\text {finite }}=\frac{D_{4}\left[g \rightarrow Q \bar{Q}\left({ }^{3} S_{1}^{[1]}\right)\right]^{\text {finite }}}{\left\langle 0\left|\mathcal{O}_{4}^{Q \bar{Q}\left({ }^{3} S_{1}^{[1]}\right)}\left({ }^{3} S_{1}^{[1]}\right)\right| 0\right\rangle^{(0)}}=\frac{D_{4}\left[g \rightarrow Q \bar{Q}\left({ }^{3} S_{1}^{[1]}\right)\right]^{\text {finite }}}{2(d-1) N_{c} \boldsymbol{q}^{4}}
$$

where $D_{4}\left[g \rightarrow Q \bar{Q}\left({ }^{3} S_{1}^{[1]}\right)\right]^{\text {finite }}$ and $\left\langle 0\left|\mathcal{O}_{4}^{Q \bar{Q}\left({ }^{3} S_{1}^{[1]}\right)}\left({ }^{3} S_{1}^{[1]}\right)\right| 0\right\rangle^{(0)}$ are given in Eqs. (5.34) and (6.1e), respectively. The result in Eq. (7.7) is new. As expected, both the double and single poles in $\epsilon_{\mathrm{IR}}$ have cancelled in Eq. (7.7). These cancellations rely nontrivially on the correctness of the infrared subtractions and on the NRQCD operator renormalizations in our calculation.

Finally, we note that the color-singlet short-distance coefficient $d_{0}\left[g \rightarrow Q \bar{Q}\left({ }^{1} S_{0}^{[1]}\right)\right]{ }^{(2)}$ was computed in Ref. [29]. We can obtain the corresponding color-octet short-distance coefficient by multiplying by the ratio of the color-octet and color-singlet color factors, namely, $\left[\left(N_{c}^{2}-1\right) /\left(4 N_{c}^{2}\right)\right]_{N_{c}=3}^{-1}\left[\left(N_{c}^{2}-4\right) /\left(4 N_{c}\right)\right]$. Then, we find that

$$
d_{0}\left[g \rightarrow Q \bar{Q}\left({ }^{1} S_{0}^{[8]}\right)\right]^{(2)}=\frac{\alpha_{s}^{2}}{8 m^{3}} \frac{N_{c}^{2}-4}{4 N_{c}}\left[3 z-2 z^{2}+2(1-z) \log (1-z)\right] .
$$

\section{Numerical results}

In this section we describe the numerical results that derive from our calculations.

We have evaluated $d_{4}\left[g \rightarrow Q \bar{Q}\left({ }^{3} S_{1}^{[1]}\right)\right]^{\text {finite }}(z)$ in Eq. (7.8) by carrying out the integrations over the phase space $d \tilde{\Phi}_{2}$ in Eqs. (5.32), (5.35a), and (5.35b) numerically at 100 points in each of the ranges $z=0$ to $z=10^{-2}, z=10^{-2}$ to $z=1-10^{-2}$, and $z=1-10^{-2}$ to $z=1$. We have then used the parametrization in Eq. (B.2) to obtain a best fit to the numerical results, which leads to the parameters that are given in Table 3. Details of this procedure are given in Appendix B. We have also applied this procedure to $d_{0}\left[g \rightarrow Q \bar{Q}\left({ }^{3} S_{1}^{[1]}\right)\right]^{(3)}(z)$ and $d_{2}\left[g \rightarrow Q \bar{Q}\left({ }^{3} S_{1}^{[1]}\right)\right]^{(3)}(z)$. In Fig. 8, we plot the results of the numerical calculations of the short-distance coefficients. 


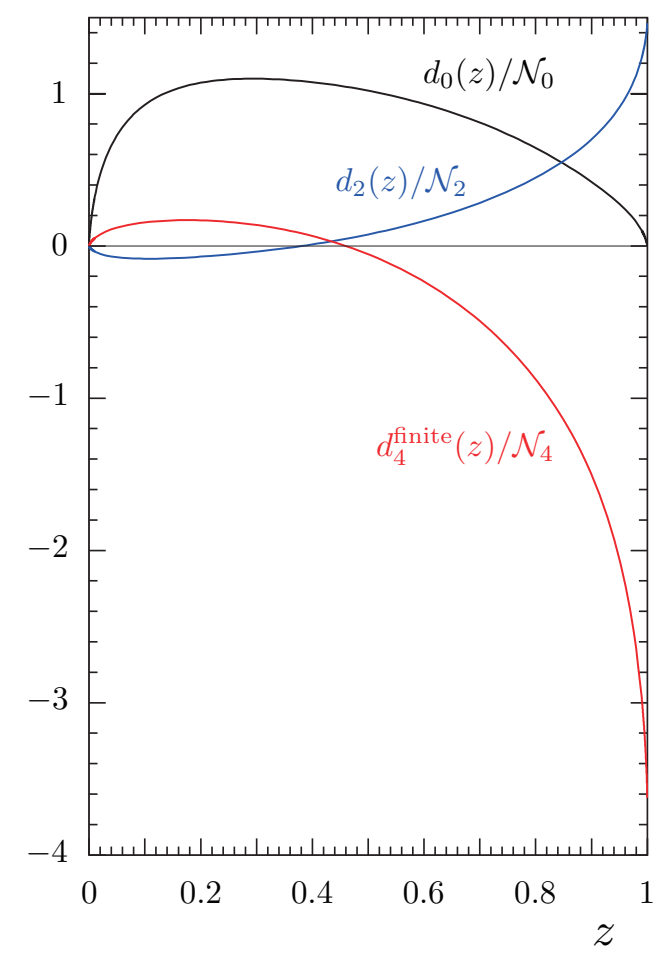

Figure 8. The color-singlet short-distance coefficients $d_{0}\left[g \rightarrow Q \bar{Q}\left({ }^{3} S_{1}^{[1]}\right)\right]^{(3)}(z), \quad d_{2}[g \rightarrow$ $\left.Q \bar{Q}\left({ }^{3} S_{1}^{[1]}\right)\right]{ }^{(3)}(z)$ and $d_{4}\left[g \rightarrow Q \bar{Q}\left({ }^{3} S_{1}^{[1]}\right)\right]^{\text {finite }}(z)$, which are defined in Eqs. (3.11), (3.12) and (7.7), respectively, as functions of $z$. The scaling factors are $\left(\mathcal{N}_{0}, \mathcal{N}_{2}, \mathcal{N}_{4}\right)=\left(10^{-3} \times \alpha_{s}^{3} / m^{3}, 10^{-2} \times\right.$ $\left.\alpha_{s}^{3} / m^{5}, 10^{-2} \times \alpha_{s}^{3} / m^{7}\right)$.

We would like to obtain estimates of the relative sizes of the contributions of the various fragmentation functions to the cross section for $J / \psi$ production in hadron-hadron collisions. The contribution of a fragmentation process to the $J / \psi$ production cross section, differential in the $J / \psi$ transverse momentum $p_{T}$, is

$$
\frac{d \sigma_{J / \psi}^{\mathrm{frag}}}{d p_{T}}=\int_{0}^{1} d z \frac{d \sigma_{g}}{d p_{T}}\left(p_{T} / z\right) D(z),
$$

where $\frac{d \sigma_{g}}{d p_{T}}\left(p_{T}\right)$ is the cross section to produce a gluon with transverse momentum $p_{T}$. Let us assume that $\frac{d \sigma_{g}}{d p_{T}}\left(p_{T}\right) \propto 1 / p_{T}^{\kappa}$, where $\kappa$ is a fixed power. Then,

$$
\frac{d \sigma_{J / \psi}^{\mathrm{frag}}}{d p_{T}} \propto I_{\kappa}(D),
$$

where

$$
I_{\kappa}(D)=\int_{0}^{1} d z z^{\kappa} D(z)
$$


Table 1. Results of computing $I_{\kappa}(d)$ for the order- $v^{0}$, order- $v^{2}$, and order- $v^{4}$ fragmentation functions for the fragmentation process $g \rightarrow Q \bar{Q}\left({ }^{3} S_{1}^{[1]}\right)$. The first two rows are the normalization integral $I_{0}(d)$ for the short-distance coefficients $d_{0}\left[g \rightarrow Q \bar{Q}\left({ }^{3} S_{1}^{[1]}\right)\right]^{(3)}\left[\right.$ Eq. (3.11)], $d_{2}\left[g \rightarrow Q \bar{Q}\left({ }^{3} S_{1}^{[1]}\right)\right]^{(3)}$ [Eq. (3.12)] and $d_{4,1}\left[g \rightarrow Q \bar{Q}\left({ }^{3} S_{1}^{[1]}\right)\right]^{(3)}+d_{4,2}\left[g \rightarrow Q \bar{Q}\left({ }^{3} S_{1}^{[1]}\right)\right]^{(3)}$ [Eq. (7.7)]. The third and fourth rows are $I_{0}(d)$ times the ratio of LDMEs $R_{n} \equiv\left\langle 0\left|\mathcal{O}_{n}\left({ }^{3} S_{1}^{[1]}\right)\right| 0\right\rangle /\left\langle 0\left|\mathcal{O}_{0}\left({ }^{3} S_{1}^{[1]}\right)\right| 0\right\rangle$, as is described in the text. The fifth and sixth rows are the integral $I_{5.2}(d)$. The seventh and eighth rows are $I_{5.2}(d)$ times $R_{n}$. In the case of $d_{4,1}\left[g \rightarrow Q \bar{Q}\left({ }^{3} S_{1}^{[1]}\right)\right]^{(3)}+d_{4,2}\left[g \rightarrow Q \bar{Q}\left({ }^{3} S_{1}^{[1]}\right)\right]^{(3)}$, we have set $\mu_{\Lambda}=m$ and $2 m$. The quantities in the table are divided by the factor $\mathcal{N}=10^{-4} \times \alpha_{s}^{3} / \mathrm{m}^{3}$.

\begin{tabular}{|clcc|}
\hline$I_{\kappa}\left(d_{n}\right) \backslash d_{n}\left[g \rightarrow Q \bar{Q}\left({ }^{3} S_{1}^{[1]}\right)\right]^{(3)}$ & $d_{0}$ & $d_{2}$ & $d_{4,1}+d_{4,2}$ \\
\hline$\left.I_{0}\left(d_{n}\right)\right|_{\mu_{\Lambda}=m}$ & 8.29 & $20.3 / m^{2}$ & $-37.4 / m^{4}$ \\
$\left.I_{0}\left(d_{n}\right)\right|_{\mu_{\Lambda}=2 m}$ & 8.29 & $20.3 / m^{2}$ & $-6.54 / m^{4}$ \\
$\left.I_{0}\left(d_{n}\right)\right|_{\mu_{\Lambda}=m} \times R_{n}$ & 8.29 & 3.95 & -1.41 \\
$\left.I_{0}\left(d_{n}\right)\right|_{\mu_{\Lambda}=2 m} \times R_{n}$ & 8.29 & 3.95 & -0.247 \\
\hline$\left.I_{5.2}\left(d_{n}\right)\right|_{\mu_{\Lambda}=m}$ & 0.743 & $11.2 / m^{2}$ & $35.7 / m^{4}$ \\
$\left.I_{5.2}\left(d_{n}\right)\right|_{\mu_{\Lambda}=2 m}$ & 0.743 & $11.2 / m^{2}$ & $84.5 / m^{4}$ \\
$\left.I_{5.2}\left(d_{n}\right)\right|_{\mu_{\Lambda}=m} \times R_{n}$ & 0.743 & 2.18 & 1.35 \\
$\left.I_{5.2}\left(d_{n}\right)\right|_{\mu_{\Lambda}=2 m} \times R_{n}$ & 0.743 & 2.18 & 3.19 \\
\hline
\end{tabular}

Hence, we can obtain a rough estimate of the relative contribution of a fragmentation process to the cross section by computing $I_{\kappa}(D) .{ }^{7}$

We can estimate $\kappa$ by taking advantage of the fact that $J / \psi$ production in the ${ }^{3} S_{1}^{[8]}$ channel is dominated at LO in $\alpha_{s}$ at large $p_{T}$ by gluon fragmentation into a $J / \psi$ with longitudinal-momentum fraction $z=1$ (Ref. [45]). Consequently, the $p_{T}$ dependence in this channel at high $p_{T}$ is approximately that of $d \sigma_{g} / d p_{T}$. Furthermore, since the NLO $k$ factor for this channel is essentially independent of $p_{T}$ and amounts to a correction of only about $14 \%$ at the Tevatron [46], we do not expect the NLO corrections to change the $p_{T}$ dependence of this channel significantly. Therefore, we estimate $\kappa$ by making use of the result for the ${ }^{3} S_{1}^{[8]}$ contribution to $d \sigma / d p_{T}$ at NLO in $\alpha_{s}$ that appears in Fig. 1(c) of Ref. [7]. Specifically, we compute $\left(d / d \log p_{T}\right) \log \left[d \sigma / d p_{T}\right]$ and find that $\kappa \approx 5.2$ at $p_{T}=20 \mathrm{GeV}$.

We have computed $I_{\kappa}(d)$ for $\kappa=0$ and 5.2 , taking $d$ to be the order- $v^{0}$, order- $v^{2}$ and order- $v^{4}$ fragmentation functions for the fragmentation process $g \rightarrow Q \bar{Q}\left({ }^{3} S_{1}^{[1]}\right)$. The results are shown in Table 1. In the first and second rows of Table 1, we give the normalization integrals $I_{0}(d)$ for the short-distance coefficients. In the third and fourth rows, we give $I_{0}(d)$ multiplied by the relative value of corresponding LDME. In the fifth and sixth rows we give $I_{5.2}(d)$. In the seventh and eighth rows we give $I_{5.2}(d)$ multiplied by the relative value of corresponding LDME. We take the relative values of the LDMEs to be given by

\footnotetext{
${ }^{7}$ The precise calculation of a fragmentation contribution to a cross section would require that one compute the convolution of the two-to-two partonic cross sections that produce a final-state gluon with the fragmentation function and with the appropriate parton distributions. Such a calculation is beyond the scope of the present paper.
} 
the generalized Gremm-Kapustin relation [28]:

$$
\left\langle 0\left|\mathcal{O}_{0}^{J / \psi}\left({ }^{3} S_{1}^{[1]}\right)\right| 0\right\rangle:\left\langle 0\left|\mathcal{O}_{2}^{J / \psi}\left({ }^{3} S_{1}^{[1]}\right)\right| 0\right\rangle:\left\langle 0\left|\mathcal{O}_{4}^{J / \psi}\left({ }^{3} S_{1}^{[1]}\right)\right| 0\right\rangle=1: m^{2}\left\langle v^{2}\right\rangle: m^{4}\left\langle v^{2}\right\rangle^{2} .
$$

We use the value of $m^{2}\left\langle v^{2}\right\rangle$ from Table I of Ref. [47]:

$$
m^{2}\left\langle v^{2}\right\rangle=0.437 \mathrm{GeV}^{2} \text { at } m=1.5 \mathrm{GeV} .
$$

Examining the relative values in the fifth or sixth rows of Table 1, we see that, if we exclude the factors from the NRQCD LDMEs, then the order- $v^{4}$ contribution is enhanced considerably relative to the order- $v^{0}$ and order- $v^{2}$ contributions. We attribute this enhancement to the strong peaking of $d_{4,1}\left[g \rightarrow Q \bar{Q}\left({ }^{3} S_{1}^{[1]}\right)\right]^{(3)}+d_{4,2}\left[g \rightarrow Q \bar{Q}\left({ }^{3} S_{1}^{[1]}\right)\right]^{(3)}$ near $z=1$, which arises primarily from the $\delta$ functions and + distributions that it contains. We expect such strong peaking to occur whenever the full-QCD process contains soft divergences that correspond to divergences in the $Q \bar{Q}$ LDMEs.

From the seventh and eighth rows of Table 1, we see that the order- $v^{4}$ fragmentation contribution to the cross section is enhanced by approximately a factor of 2 for $\mu_{\Lambda}=m$ and by approximately a factor of 4 for $\mu_{\Lambda}=2 m$, relative to the order- $v^{0}$ contribution. However, the order- $v^{0}$ contribution to the $J / \psi$ cross section at $p_{T}=10 \mathrm{GeV}$ lies about a factor of 30 below the measured cross section. Thus, while the enhancement of the order- $v^{4}$ fragmentation contribution to the cross section is substantial, it is not sufficient to make the order- $v^{4}$ fragmentation contribution to the cross section important for the phenomenology of $J / \psi$ production at the current level of precision.

We can also compare the relative contributions to $d \sigma_{J / \psi}^{\mathrm{frag}}$ of each of the three $Q \bar{Q}$ channels in that appear in $D[g \rightarrow J / \psi]$ in order $v^{4}$ [Eq. (3.14)]. For each channel, we compute $I_{\kappa}(5.2)$. Then, we multiply by the following LDMEs:

$$
\begin{aligned}
& \left\langle 0\left|\mathcal{O}_{4}^{J / \psi}\left({ }^{3} S_{1}^{[1]}\right)\right| 0\right\rangle=m^{4}\left\langle v^{2}\right\rangle^{2} \times 1.32 \mathrm{GeV}^{3} \\
& \left\langle 0\left|\mathcal{O}_{0}^{J / \psi}\left({ }^{3} P^{[8]}\right)\right| 0\right\rangle=-0.109 \mathrm{GeV}^{5} \\
& \left\langle 0\left|\mathcal{O}_{0}^{J / \psi}\left({ }^{3} S_{1}^{[8]}\right)\right| 0\right\rangle=3.12 \times 10^{-3} \mathrm{GeV}^{3} \\
& \left\langle 0\left|\mathcal{O}_{0}^{J / \psi}\left({ }^{1} S_{0}^{[8]}\right)\right| 0\right\rangle=4.50 \times 10^{-2} \mathrm{GeV}^{3}
\end{aligned}
$$

where $m^{2}\left\langle v^{2}\right\rangle$ is given in Eq. (8.5), and the numerical values on the right sides of Eq. (8.6) come from the fit at NLO in $\alpha_{s}$ to the Tevatron and HERA data in Ref. [7]. The results of this computation are shown in Table 2 .

We see from the second row of Table 2 that the $Q \bar{Q}\left({ }^{3} S_{1}^{[1]}\right)$ channel makes a small contribution to $D_{4}[g \rightarrow J / \psi]$ at $p_{T}=20 \mathrm{GeV}$, confirming our previous conclusion that this channel is not important phenomenologically at the current level of precision. We also see that the $Q \bar{Q}\left({ }^{3} S_{1}^{[8]}\right)$ and $Q \bar{Q}\left({ }^{3} P^{[8]}\right)$ channels give comparable contributions to $D_{4}[g \rightarrow J / \psi]$ at $p_{T}=20 \mathrm{GeV}$. As we have mentioned, at high $p_{T}$ at LO in $\alpha_{s}$, the fragmentation contribution gives the bulk of the contribution in the $Q \bar{Q}\left({ }^{3} S_{1}^{[8]}\right)$ channel (Ref. [45]). As we have also mentioned, the correction to the production rate in the $Q \bar{Q}\left({ }^{3} S_{1}^{[8]}\right)$ channel at NLO in $\alpha_{s}$ is only about $14 \%$ at the Tevatron [46]. Hence, we can estimate the fragmentation contribution to $J / \psi$ production at the Tevatron in the $Q \bar{Q}\left({ }^{3} P^{[1]}\right)$ channel by multiplying 
Table 2. Relative contributions to $d \sigma_{J / \psi}^{\mathrm{frag}}$ in order $v^{4}$. The first and second rows give $I_{0}(d)$ for the short-distance coefficients in Eq. (3.14). The second and third rows give $I_{0}(d)$ times the LDMEs in Eq. (8.6). The fourth and fifth rows give $I_{5.2}(d)$. The fifth and sixth rows give $I_{5.2}(d)$ times the LDMEs in Eq. (8.6). We take $m=m_{c}=1.5 \mathrm{GeV}$. For compatibility with Ref. [7], we take $\alpha_{s}=\alpha_{s}\left(m_{T}\right)$, where $m_{T}=\sqrt{p_{T}^{2}+4 m_{c}^{2}}$. We choose the point $p_{T}=20 \mathrm{GeV}$, which implies that $\alpha_{s}\left(m_{T}\right)=0.154$.

\begin{tabular}{|ccccl|}
\hline$I_{\kappa}(d) \backslash$ channel & $\mathcal{O}_{0}^{J / \psi}\left({ }^{1} S_{0}^{[8]}\right)$ & $\mathcal{O}_{0}^{J / \psi}\left({ }^{3} S_{1}^{[8]}\right)$ & $\mathcal{O}_{0}^{J / \psi}\left({ }^{3} P^{[8]}\right)$ & $\mathcal{O}_{4}^{J / \psi}\left({ }^{3} S_{1}^{[1]}\right)$ \\
\hline$\left.I_{0}(d)\right|_{\mu_{\Lambda}=m} \times 10^{6} \mathrm{GeV}^{3}$ & 122. & 5970 & $-171 . \mathrm{GeV}^{-2}$ & $-0.799 \mathrm{GeV}^{-4}$ \\
$\left.I_{0}(d)\right|_{\mu_{\Lambda}=2 m} \times 10^{6} \mathrm{GeV}^{3}$ & 122. & 5970 & $-271 . \mathrm{GeV}^{-2}$ & $-0.140 \mathrm{GeV}^{-4}$ \\
$\left.I_{0}(d)\right|_{\mu_{\Lambda}=m} \times 10^{6} \mathrm{LDME}$ & 5.49 & 18.6 & 18.6 & -0.201 \\
$\left.I_{0}(d)\right|_{\mu_{\Lambda}=2 m} \times 10^{6} \mathrm{LDME}$ & 5.49 & 18.6 & 29.6 & -0.0353 \\
\hline$\left.I_{5.2}(d)\right|_{\mu_{\Lambda}=m} \times 10^{6} \mathrm{GeV}^{3}$ & 36.7 & 5970 & $-300 . \mathrm{GeV}^{-2}$ & $0.763 \mathrm{GeV}^{-4}$ \\
$\left.I_{5.2}(d)\right|_{\mu_{\Lambda}=2 m} \times 10^{6} \mathrm{GeV}^{3}$ & 36.7 & 5970 & $-400 . \mathrm{GeV}^{-2}$ & $1.81 \mathrm{GeV}^{-4}$ \\
$\left.I_{5.2}(d)\right|_{\mu_{\Lambda}=m} \times 10^{6} \mathrm{LDME}$ & 1.65 & 18.6 & 32.7 & 0.192 \\
$\left.I_{5.2}(d)\right|_{\mu_{\Lambda}=2 m} \times 10^{6} \mathrm{LDME}$ & 1.65 & 18.6 & 43.6 & 0.455 \\
\hline
\end{tabular}

the ratio of the $Q \bar{Q}\left({ }^{3} P^{[8]}\right)$ and $Q \bar{Q}\left({ }^{3} S_{1}^{[8]}\right)$ entries in the seventh row of Table 2 by the value of $d \sigma / d p_{T} \times B(J / \psi \rightarrow \mu \mu)$ at $20 \mathrm{GeV}$ from Fig. 1(c) of Ref. [7] and by dividing by 1.14 to account for the NLO correction in the $Q \bar{Q}\left({ }^{3} S_{1}^{[8]}\right)$ channel. Our estimate is that the fragmentation contribution to $d \sigma / d p_{T} \times B(J / \psi \rightarrow \mu \mu)$ at $p_{T}=20 \mathrm{GeV}$ from the $Q \bar{Q}\left({ }^{3} P^{[8]}\right)$ channel is about $6 \times 10^{-3} \mathrm{nb} / \mathrm{GeV}$. We see that this is comparable to (about a factor of 2 larger than) the total NLO contribution in the $Q \bar{Q}\left({ }^{3} P^{[8]}\right)$ channel in Fig. 1(c) of Ref. [7]. A more precise calculation of the fragmentation contribution in the $Q \bar{Q}\left({ }^{3} P^{[8]}\right)$ channel will be necessary in order to determine whether it is the dominant contribution in that channel at NLO in $\alpha_{s}$ at high $p_{T}$.

\section{Summary}

We have calculated NRQCD short-distance coefficients for gluon fragmentation into a ${ }^{3} S_{1}$ heavy-quarkonium state through relative order $v^{4}$. Our principal new result is the expression for the sum of the relative-order- $v^{4}$ short-distance coefficients $d_{4,1}\left[g \rightarrow Q \bar{Q}\left({ }^{3} S_{1}^{[1]}\right)\right]^{(3)}+$ $d_{4,2}\left[g \rightarrow Q \bar{Q}\left({ }^{3} S_{1}^{[1]}\right)\right]^{(3)}$ for gluon fragmentation through the ${ }^{3} S_{1}$ color-singlet channel. This expression is given in Eq. (7.7) and in the parametrization of $d_{4}\left[g \rightarrow Q \bar{Q}\left({ }^{3} S_{1}^{[1]}\right)\right]^{\text {finite }}$ in Eq. (B.2). As a byproduct of this calculation, we have computed the short-distance coefficient for gluon fragmentation through the ${ }^{3} S_{1}$ color-octet channel [Eq. (7.4)], finding agreement with the results in Refs. [30,31], and the sum of short-distance coefficients for gluon fragmentation through the ${ }^{3} P_{J}$ color-octet channel [Eq. (7.5)], finding agreement with the result in Ref. [29] for the ${ }^{3} P_{J}$ color-singlet short-distance coefficients. We have also computed the short-distance coefficients for gluon fragmentation through the ${ }^{3} S_{1}$ color- 
singlet channel at leading order in $v$ and at relative-order $v^{2}$ and find agreement with the results in Refs. [19, 20] and Ref. [21], respectively.

The analysis in this paper involves, for the first time in an NRQCD factorization calculation, both single and double soft divergences in the full-QCD process. These soft divergences manifest themselves as single and double poles in $\epsilon=(4-d) / 2$ in dimensional regularization. We have dealt with the soft divergences by devising subtractions that remove both the single and double poles in $\epsilon$. We have calculated the phase-space integrals for the subtraction contributions analytically in $d=4-2 \epsilon$ dimensions and have calculated the phase-space integrals for the finite remainder contributions numerically in $d=4$ dimensions. We have also calculated the perturbative NRQCD LDMEs that appear in the NRQCD matching equations for the short-distance coefficients. These perturbative LDMEs involve both one-loop and two-loop renormalizations of the NRQCD operators. Our results for the renormalization-group evolution of the renormalized LDMEs confirm some results in Refs. [2, 23, 44], but disagree with one of the results in Ref. [23].

In Tables 1 and 2, we have given estimates of the relative sizes of the contributions of the various channels to gluon fragmentation into $J / \psi$ through relative order $v^{4}$. As we have mentioned, the contribution of the ${ }^{3} S_{1}$ color-octet channel is believed to be important phenomenologically at large $p_{T}$. Hence, one might expect the order- $v^{4}$ contribution in the ${ }^{3} S_{1}$ color-singlet channel to be important as well, since the color-singlet channel mixes with the color-octet channel through single and double logarithms of the NRQCD factorization scale at order $v^{4}$. Indeed, we find that the contribution to the cross section at $p_{T}=20 \mathrm{GeV}$ of the ${ }^{3} S_{1}$ color-singlet channel in order $v^{4}$ is about a factor of 2 larger than the contribution of the ${ }^{3} S_{1}$ color-singlet channel at the leading order in $v$ when the factorization scale is taken to be $\mu_{\Lambda}=m_{c}$ and is about a factor of 4 larger than the contribution of the ${ }^{3} S_{1}$ color-singlet channel at the leading order in $v$ when the factorization scale is taken to be $\mu_{\Lambda}=2 m_{c}$. This is a large enhancement, since one would nominally expect the order- $v^{4}$ contribution to be about $\left\langle v^{2}\right\rangle^{2} \approx 0.04$ times the order- $v^{0}$ contribution. In spite of this large enhancement of the fragmentation contribution in order $v^{4}$, the corresponding contribution to the $J / \psi$ production cross section at the Tevatron or the LHC is not important at the current level of precision of the phenomenology.

We attribute the large enhancement of the order- $v^{4}$ contribution to the ${ }^{3} S_{1}$ colorsinglet channel to the peaking of the sum of short-distance coefficients near $z=1$. This peaking arises from terms that are proportional to $\delta(1-z),[1 /(1-z)]_{+}$, and $[\log (1-$ $z) /(1-z)]_{+}$. These terms are remnants of the soft divergences that appear in the fullQCD expression for the fragmentation process and that, ultimately, cancel in the NRQCD matching equations for the short-distance coefficients. We expect such a peaking, and the corresponding enhancement, to be present whenever soft divergences appear in a full-QCD process and are cancelled in the NRQCD matching equations. ${ }^{8}$

The signature of this peaking/enhancement in Table 2 is that the magnitude of the

\footnotetext{
${ }^{8}$ There is also some peaking/enhancement in the order- $v^{2}$ contribution to the ${ }^{3} S_{1}$ color-singlet channel. The order $-v^{2}$ contribution contains terms that are the product of an order- $v^{0}$ amplitude with an order- $v^{2}$ amplitude. The order- $v^{2}$ amplitude is sufficiently singular that its square would produce a soft divergence. However, in combination with the order- $v^{0}$ amplitude, it produces only a peaking at $z=1$.
} 
$I_{5.2}$ entry is comparable to or greater than the magnitude of the $I_{0}$ entry. We see this signature of peaking/enhancement in the ${ }^{3} S_{1}$ and ${ }^{3} P_{J}$ color-octet channels, as well as in the ${ }^{3} S_{1}$ color-singlet channel in order $v^{4}$, but not in the ${ }^{1} S_{0}$ color-octet channel. The ${ }^{3} P_{J}$ color-octet channel contains soft divergences in the full-QCD process, but the ${ }^{1} S_{0}$ coloroctet channel does not. (The fragmentation contribution in the ${ }^{3} S_{1}$ color-octet channel is proportional to $\delta(1-z)$ at leading order in $\alpha_{s}$.)

The fragmentation contribution to the ${ }^{3} S_{1}$ color-octet channel dominates the contribution of that channel to the $J / \psi$ production cross section at hadron-hadron colliders at large $p_{T}$, both at LO in $\alpha_{s}$ (Ref. [45]) and at NLO in $\alpha_{s}$ (Ref. [46]). We estimate that the fragmentation contribution to the ${ }^{3} P_{J}$ color-octet channel gives a substantial part of the total contribution of that channel at NLO in $\alpha_{s}$ to the $J / \psi$ production cross section at hadron-hadron colliders at large $p_{T}$. A more precise calculation of the ${ }^{3} P_{J}$ color-octet fragmentation contribution will be required in order to determine if it is the dominant contribution at large $p_{T}$ in that channel.

\section{Acknowledgments}

We thank Zhi-Guo He for providing us with his unpublished results for the renormalizationgroup evolution of NRQCD LDMEs. We also thank Andrea Petrelli for the use of some of his mathematica code. The work of G.T.B. in the High Energy Physics Division at Argonne National Laboratory was supported by the U. S. Department of Energy, Division of High Energy Physics, under Contract No. DE-AC02-06CH11357. The work of U.R.K. and J.L. was supported by the MEST of Korea under the NRF Grants No. 2011-0027559 and No. 2011-0003023, respectively.

\section{A Analytic calculation of phase-space integrals}

In this appendix, we give some of the details of the analytic integrations over the final-state phase space for $S_{12}, S_{1}$ and $S_{2}$.

We first carry out the average over the angles of the transverse components of the final-state gluon momenta in the phase-space (4.8c). Under this angular averaging we have

$$
\begin{aligned}
\langle x\rangle_{\perp} & =\frac{z_{1} z_{2}}{z}\left(\frac{e_{1}}{z_{1}}+\frac{e_{2}}{z_{2}}-\frac{1}{z}\right) \\
\left\langle x^{2}\right\rangle_{\perp} & =\frac{z_{1}^{2} z_{2}^{2}}{z^{2}}\left(\frac{e_{1}}{z_{1}}+\frac{e_{2}}{z_{2}}-\frac{1}{z}\right)^{2}+\frac{z_{1} z_{2}}{(d-2) z^{2}}\left(2 e_{1}-\frac{z_{1}}{z}\right)\left(2 e_{2}-\frac{z_{2}}{z}\right) .
\end{aligned}
$$

After this angular averaging, the squared amplitude is independent of the directions of $\overline{\boldsymbol{k}}_{1 \perp}$ and $\overline{\boldsymbol{k}}_{2 \perp}$. 
In evaluating the integrals over $\left|\overline{\boldsymbol{k}}_{1 \perp}\right|$ and $\left|\overline{\boldsymbol{k}}_{2 \perp}\right|$, it is convenient to define dimensionless variables $u_{1}$ and $u_{2}$ :

$$
\begin{aligned}
& u_{1}=\left(\frac{z}{z_{1}}\right)^{2} \bar{k}_{1 \perp}^{2}, \\
& u_{2}=\left(\frac{z}{z_{2}}\right)^{2} \bar{k}_{2 \perp}^{2} .
\end{aligned}
$$

Then $e_{1}, e_{2}$ and $x$ can be expressed as

$$
\begin{aligned}
& e_{1}=\frac{z_{1}}{2 z}\left(1+u_{1}\right), \\
& e_{2}=\frac{z_{2}}{2 z}\left(1+u_{2}\right) .
\end{aligned}
$$

The integrals of $S_{12}, S_{1}$ and $S_{2}$ over the magnitudes of the transverse components of the final-state gluon momenta can be expressed as linear combinations of the following elementary integrals:

$$
\begin{aligned}
J_{m n}^{i} & =\int \frac{d^{d-2} \overline{\boldsymbol{k}}_{i \perp}}{(2 \pi)^{d-2}} \frac{1}{e_{i}^{m}\left(1+2 e_{i}\right)^{n}} \\
& =\frac{2^{m}}{(4 \pi)^{1-\epsilon} \Gamma(1-\epsilon)}\left(\frac{z_{i}}{z}\right)^{2-m-n-2 \epsilon} \int_{0}^{\infty} \frac{d u_{i}}{u_{i}^{\epsilon}\left(1+u_{i}\right)^{m}\left(1+\frac{z}{z_{i}}+u_{i}\right)^{n}},
\end{aligned}
$$

where $m$ takes on integer values equal to or greater than -1 , and $n$ takes on non-negative integer values. These integrals can be evaluated straightforwardly to obtain

$$
\begin{aligned}
J_{m 0}^{i}= & 2^{m}\left(\frac{z_{i}}{z}\right)^{2-m-2 \epsilon} \frac{\Gamma(m-1+\epsilon)}{(4 \pi)^{1-\epsilon} \Gamma(m)} \\
J_{0 n}^{i}= & \left(\frac{z_{i}}{z}\right)^{1-\epsilon}\left(1+\frac{z_{i}}{z}\right)^{1-n-\epsilon} \frac{\Gamma(n-1+\epsilon)}{(4 \pi)^{1-\epsilon} \Gamma(n)} \\
J_{-1,2}^{i}= & \frac{1}{2}\left(J_{01}^{i}-J_{02}^{i}\right), \\
J_{m n}^{i}= & (-1)^{m+n}\left(\frac{z_{i}}{z}\right)^{1-\epsilon} \frac{\Gamma(\epsilon)}{(4 \pi)^{1-\epsilon} \Gamma(m) \Gamma(n)} \\
& \times\left.\left(\frac{\partial}{\partial a}\right)^{m-1}\left(\frac{\partial}{\partial b}\right)^{n-1}\left\{\frac{2}{b-2 a}\left[\left(2 a+\frac{z_{i}}{z}\right)^{-\epsilon}-\left(b+\frac{z_{i}}{z}\right)^{-\epsilon}\right]\right\}\right|_{a=0, b=1} .
\end{aligned}
$$

After we have carried out the integrations over the transverse components of the final-state gluon momenta, the remaining integrals over $z_{1}$ and $z_{2}$ for $S_{12}$ are simple. The integrations over $z_{1}$ and $z_{2}$ for $S_{1}$ and $S_{2}$ can be expressed as linear combinations of the integrals

$$
\begin{aligned}
A_{n} & =\int_{z_{1} z_{2}} \frac{z_{2}{ }^{n-\epsilon}}{z_{1}^{1+2 \epsilon}\left(z+z_{2}\right)^{1+\epsilon}}, \\
B_{n} & =\int_{z_{1} z_{2}} \frac{z_{2}{ }^{n-\epsilon}}{z_{1}^{1+2 \epsilon}\left(z+z_{2}\right)^{\epsilon}}, \\
C_{n} & =\int_{z_{1} z_{2}} \frac{z_{2}{ }^{n-2 \epsilon}}{z_{1}^{1+2 \epsilon}} \\
D_{n} & =\int_{z_{1} z_{2}} \frac{z_{2}{ }^{n-2 \epsilon}}{z_{1}^{1+2 \epsilon}\left(z+z_{2}\right)},
\end{aligned}
$$


where

$$
\int_{z_{1} z_{2}} F\left(z_{1}, z_{2}\right)=\int_{0}^{1} d z_{1} \int_{0}^{1} d z_{2} F\left(z_{1}, z_{2}\right) \delta\left(1-z-z_{1}-z_{2}\right) .
$$

By making use of the identity $z_{2} /\left(z+z_{2}\right)=1-z /\left(z+z_{2}\right)$, we derive the relations

$$
\begin{aligned}
& A_{n}=B_{n-1}-z A_{n-1}, \\
& D_{n}=C_{n-1}-z D_{n-1} .
\end{aligned}
$$

Applying the recursion relations (A.8) repeatedly, we can reduce all of the integrals to the forms $A_{0}, B_{n}$ and $C_{n}$, with $1 \leq n \leq 4$. It turns out that the coefficient of $D_{0}$ vanishes. The coefficient of $A_{0}$ is of order $\epsilon^{0}$, while the coefficients of the $B_{n}$ are of order $\epsilon^{-1}$ or $\epsilon^{0}$. Therefore, we evaluate $A_{0}$ only through order $\epsilon^{0}$, and we evaluate the $B_{n}$ only through order $\epsilon$. The expressions for $A_{0}, B_{n}$ and $C_{n}$ can be obtained conveniently by making use of the identity

$$
\frac{1}{z_{1}^{1+2 \epsilon}}=-\frac{(1-z)^{-2 \epsilon}}{2 \epsilon} \delta\left(z_{1}\right)+\left[\frac{1}{z_{1}^{1+2 \epsilon}}\right]_{1-z},
$$

which applies when the domain of integration is $0 \leq z_{1} \leq 1-z$. The distribution in the second term of Eq. (A.9) is defined by

$$
\int_{0}^{1-z} d z_{1} f\left(z_{1}\right)\left[\frac{1}{z_{1}^{1+2 \epsilon}}\right]_{1-z}=\int_{0}^{1-z} d z_{1} \frac{f\left(z_{1}\right)-f(0)}{z_{1}^{1+2 \epsilon}} .
$$

A straightforward evaluation of the integrals then gives

$$
\begin{aligned}
A_{0} & =-\frac{1}{2 \epsilon(1-z)^{3 \epsilon}}-\log z+O(\epsilon) \\
B_{n} & =-\frac{(1-z)^{n-3 \epsilon}}{2 \epsilon}+(1-z)^{n-3 \epsilon}\left[X_{n}+Y_{n}+Z_{n}+O\left(\epsilon^{2}\right)\right] \\
C_{n} & =(1-z)^{n-4 \epsilon}\left\{-\frac{1}{2 \epsilon}+\frac{1}{2 \epsilon}\left[1-\frac{\Gamma(n+1-2 \epsilon) \Gamma(1-2 \epsilon)}{\Gamma(n+1-4 \epsilon)}\right]\right\},
\end{aligned}
$$

where

$$
\begin{aligned}
& X_{n}=\int_{0}^{1} d t \frac{(1-t)^{n}-1}{t}=-\sum_{k=1}^{n} \frac{1}{k} \\
& Y_{n}=-\epsilon \int_{0}^{1} d t \frac{(1-t)^{n}}{t}\{\log (1-t)+\log [1-(1-z) t]\}, \\
& Z_{n}=-2 \epsilon \int_{0}^{1} d t \frac{(1-t)^{n}-1}{t} \log t=-2 \epsilon \sum_{k=1}^{n} \frac{1}{k} \sum_{\ell=1}^{k} \frac{1}{\ell}
\end{aligned}
$$

In Eq. (A.11c), the first term in the braces is the pole contribution, and the remainder is 
finite. The results for $Y_{n}$ for $0 \leq n \leq 4$ are

$$
\begin{aligned}
& Y_{0}=\epsilon\left[\frac{\pi^{2}}{6}+\operatorname{Li}_{2}(1-z)\right] \\
& Y_{1}=\epsilon\left[\frac{\pi^{2}}{6}+\operatorname{Li}_{2}(1-z)-2-\frac{z}{1-z} \log z\right] \\
& Y_{2}=\epsilon\left[\frac{\pi^{2}}{6}+\operatorname{Li}_{2}(1-z)-\frac{5-6 z}{2(1-z)}-\frac{z(2-3 z) \log z}{2(1-z)^{2}}\right] \\
& Y_{3}=\epsilon\left[\frac{\pi^{2}}{6}+\operatorname{Li}_{2}(1-z)-\frac{49-110 z+67 z^{2}}{18(1-z)^{2}}-\frac{z\left(6-15 z+11 z^{2}\right) \log z}{6(1-z)^{3}}\right], \\
& Y_{4}=\epsilon\left[\frac{\pi^{2}}{6}+\operatorname{Li}_{2}(1-z)-\frac{205-669 z+756 z^{2}-310 z^{3}}{72(1-z)^{3}}\right. \\
&\left.-\frac{z\left(12-42 z+52 z^{2}-25 z^{3}\right) \log z}{12(1-z)^{4}}\right]
\end{aligned}
$$

\section{B Parametrizations of the short-distance coefficients}

In this appendix, we give parametrizations for the short-distance coefficients $d_{0}[g \rightarrow$ $\left.Q \bar{Q}\left({ }^{3} S_{1}^{[1]}\right)\right]^{(3)}(z), d_{2}\left[g \rightarrow Q \bar{Q}\left({ }^{3} S_{1}^{[1]}\right)\right]^{(3)}(z)$, and $d_{4}\left[g \rightarrow Q \bar{Q}\left({ }^{3} S_{1}^{[1]}\right)\right]^{\text {finite }}(z)$, which are defined in Eqs. (3.11), (3.12), and (7.8), respectively.

We observe that $d_{0}\left[g \rightarrow Q \bar{Q}\left({ }^{3} S_{1}^{[1]}\right)\right]^{(3)}(z), d_{2}\left[g \rightarrow Q \bar{Q}\left({ }^{3} S_{1}^{[1]}\right)\right]^{(3)}(z)$, and $d_{4}[g \rightarrow Q \bar{Q}$ $\left.\left({ }^{3} S_{1}^{[1]}\right)\right]^{\text {finite }}(z)$ are continuous functions of $z$ over the whole range $0 \leq z \leq 1$ and that they are not analytic at the endpoints $z=0$ and 1 . One can prove that $d_{n}(0)=0$ for $n=0,2$, and 4. In addition, $d_{n}(1)=\alpha_{s}^{3} b_{n} / m^{3+n}$ is finite and calculable analytically. The values of $b_{n}$ for $n=0,2$, and 4 are

$$
\begin{aligned}
& b_{0}=0 \\
& b_{2}=\frac{22 \pi^{2}-15}{4374 \pi}, \\
& b_{4}=-\frac{2922 \pi^{2}-2485}{229635 \pi} .
\end{aligned}
$$

Using this information, we parametrize $d_{0}\left[g \rightarrow Q \bar{Q}\left({ }^{3} S_{1}^{[1]}\right)\right]^{(3)}(z), d_{2}\left[g \rightarrow Q \bar{Q}\left({ }^{3} S_{1}^{[1]}\right)\right]^{(3)}(z)$, and $d_{4}\left[g \rightarrow Q \bar{Q}\left({ }^{3} S_{1}^{[1]}\right)\right]^{\text {finite }}(z)$ as follows:

$$
\begin{aligned}
d_{n}^{\mathrm{fit}}(z)=\frac{\alpha_{s}^{3}}{m^{3+n}}[ & b_{n} z+\log (1-z) \sum_{k=1}^{n_{a}} \alpha_{n k}(1-z)^{k}+\log ^{2}(1-z) \sum_{k=1}^{n_{b}} \beta_{n k}(1-z)^{k} \\
& \left.+\log z \sum_{k=1}^{n_{c}} \mu_{n k} z^{k}+\log ^{2} z \sum_{k=1}^{n_{d}} \nu_{n k} z^{k}+\sum_{k_{1}=1}^{n_{1}} \sum_{k_{2}=1}^{n_{2}} \omega_{n k_{1} k_{2}} z^{k_{1}}(1-z)^{k_{2}}\right] .
\end{aligned}
$$

In order to fix the parameters in Eq. (B.2), we have computed $d_{0}\left[g \rightarrow Q \bar{Q}\left({ }^{3} S_{1}^{[1]}\right)\right]^{(3)}(z)$, $d_{2}\left[g \rightarrow Q \bar{Q}\left({ }^{3} S_{1}^{[1]}\right)\right]^{(3)}(z)$, and $d_{4}\left[g \rightarrow Q \bar{Q}\left({ }^{3} S_{1}^{[1]}\right)\right]^{\text {finite }}(z)$ by numerical integration for 100 
Table 3. Results of fitting the parametrization in Eq. (B.2) to numerical values for the colorsinglet short-distance coefficients $d_{0}\left[g \rightarrow Q \bar{Q}\left({ }^{3} S_{1}^{[1]}\right)\right]^{(3)}(z), d_{2}\left[g \rightarrow Q \bar{Q}\left({ }^{3} S_{1}^{[1]}\right)\right]^{(3)}(z)$, and $d_{4}[g \rightarrow$ $\left.Q \bar{Q}\left({ }^{3} S_{1}^{[1]}\right)\right]^{\text {finite }}(z)$, which are defined in Eqs. (3.11), (3.12), and (7.8), respectively. The quantity $\Delta_{\max }^{\text {fit }}$ is described in the text.

\begin{tabular}{|crcr|}
\hline Parameters & \multicolumn{1}{c}{$d_{0}(z)$} & \multicolumn{1}{c}{$d_{2}(z)$} & \multicolumn{1}{c|}{$d_{4}(z)^{\text {finite }}$} \\
\hline$b$ & 0 & $1.4710 \times 10^{-2}$ & $-3.6531 \times 10^{-2}$ \\
\hline$\alpha_{1}$ & $-4.9866 \times 10^{-3}$ & $-1.8127 \times 10^{-2}$ & $5.2157 \times 10^{-2}$ \\
$\alpha_{2}$ & $9.8448 \times 10^{-3}$ & & $2.4588 \times 10^{-3}$ \\
$\alpha_{3}$ & $1.9512 \times 10^{-2}$ & & $3.6020 \times 10^{-2}$ \\
\hline$\beta_{1}$ & $-5.1697 \times 10^{-6}$ & $-1.2282 \times 10^{-2}$ & \\
$\beta_{2}$ & $1.0462 \times 10^{-2}$ & & $-7.3565 \times 10^{-3}$ \\
\hline$\mu_{1}$ & $-1.8921 \times 10^{-3}$ & $4.1069 \times 10^{-3}$ & $-2.5387 \times 10^{-2}$ \\
$\mu_{2}$ & & $1.8341 \times 10^{-2}$ & $-9.3477 \times 10^{-3}$ \\
$\mu_{3}$ & & & $-3.6926 \times 10^{-3}$ \\
$\mu_{4}$ & & & \\
\hline$\nu_{1}$ & $1.2154 \times 10^{-3}$ & $-1.3653 \times 10^{-3}$ & \\
$\nu_{2}$ & $1.3039 \times 10^{-3}$ & & $7.7264 \times 10^{-3}$ \\
$\nu_{3}$ & $-2.7246 \times 10^{-3}$ & & \\
$\nu_{4}$ & $-1.4814 \times 10^{-3}$ & & \\
\hline$\omega_{11}$ & $-1.6910 \times 10^{-2}$ & $-2.1832 \times 10^{-2}$ & \\
$\omega_{12}$ & $3.8110 \times 10^{-2}$ & $4.1531 \times 10^{-3}$ & \\
$\omega_{13}$ & & $8.4949 \times 10^{-4}$ & \\
$\omega_{14}$ & & $-3.8207 \times 10^{-3}$ & \\
\hline$\Delta_{\max }^{\text {fit }} \times m^{3+n} / \alpha_{s}^{3}$ & $3.26 \times 10^{-8}$ & & \\
\hline
\end{tabular}

points in each of the ranges $z=0$ to $z=10^{-2}, z=10^{-2}$ to $z=1-10^{-2}$, and $z=1-10^{-2}$ to $z=1$. We have carried out the integrations in two ways: (1) using the change of variables that was proposed in Refs. [19, 20] and is described in Appendix B of Ref. [21] and (2) using the change of variables that is given in Eq. (A.2). At each value of $z$, the numerical results from the two methods of integration agree to better than $\Delta_{n}^{\text {int }}=r_{n} d_{n}(z)$, where $\left(r_{0}, r_{2}, r_{4}\right)=\left(7.8 \times 10^{-4}, 1.7 \times 10^{-3}, 1.8 \times 10^{-3}\right)$.

We fit the parametrizations to the numerical integration results, using $\chi^{2}=\sum_{i}\left[d\left(z_{i}\right)-\right.$ $\left.d^{\text {fit }}\left(z_{i}\right)\right]^{2} /\left[\sigma\left(z_{i}\right)\right]^{2}$ as the criterion for goodness of fit, where $\sigma\left(z_{i}\right)$ is the error in the numerical integration that is given by the VEGAS integration program [48]. In cases in which it is possible to reduce the number of parameters in the fit without significantly affecting $\chi^{2}$, we have done so.

In Table 3, we show the results of this fitting procedure, along with the value for each fit of $\Delta_{\max }^{\mathrm{fit}}=\operatorname{Max}\left|d\left(z_{i}\right)-d^{\mathrm{fit}}\left(z_{i}\right)\right|$. In these fits, $\chi^{2}$ per degree of freedom is much larger than one because the error in the fit is generally considerably larger than the errors in the numerical integrations that are given by VEGAS. A conservative estimate of the overall 
error in a parametrization at each value of $z$ is $\sqrt{\left[\Delta_{\max }^{\text {fit }}\right]^{2}+\left[\Delta_{n}^{\text {int }}\right]^{2}}$.

\section{References}

[1] N. Brambilla, S. Eidelman, B.K. Heltsley, R. Vogt, G.T. Bodwin, E. Eichten, A.D. Frawley and A.B. Meyer et al., Heavy quarkonium: progress, puzzles, and opportunities, Eur. Phys. J. C 71 (2011) 1534 [arXiv:1010.5827] [InSPIRE].

[2] G.T. Bodwin, E. Braaten and G.P. Lepage, Rigorous QCD analysis of inclusive annihilation and production of heavy quarkonium, Phys. Rev. D 51 (1995) 1125 [Erratum ibid. D 55 (1997) 5853] [hep-ph/9407339] [InSPIRE].

[3] G.C. Nayak, J.-W. Qiu and G.F. Sterman, Fragmentation, factorization and infrared poles in heavy quarkonium production, Phys. Lett. B 613 (2005) 45 [hep-ph/0501235] [InSPIRE].

[4] G.C. Nayak, J.-W. Qiu and G.F. Sterman, Fragmentation, nonrelativistic QCD, and NNLO factorization analysis in heavy quarkonium production, Phys. Rev. D 72 (2005) 114012 [hep-ph/0509021] [InSPIRE].

[5] M. Butenschoen and B.A. Kniehl, Complete Next-to-Leading-Order Corrections to $J / \psi$ Photoproduction in Nonrelativistic Quantum Chromodynamics, Phys. Rev. Lett. 104 (2010) 072001 [arXiv: 0909.2798] [InSPIRE].

[6] Y.-Q. Ma, K. Wang and K.-T. Chao, $J / \psi\left(\psi^{\prime}\right)$ Production at the Tevatron and LHC at $\mathcal{O}\left(\alpha_{s}^{4} v^{4}\right)$ in Nonrelativistic QCD, Phys. Rev. Lett. 106 (2011) 042002 [arXiv:1009.3655] [InSPIRE].

[7] M. Butenschoen and B.A. Kniehl, Reconciling J/ $\psi$ Production at HERA, RHIC, Tevatron, and LHC with nonrelativistic QCD Factorization at Next-to-Leading Order, Phys. Rev. Lett. 106 (2011) 022003 [arXiv: 1009.5662] [InSPIRE].

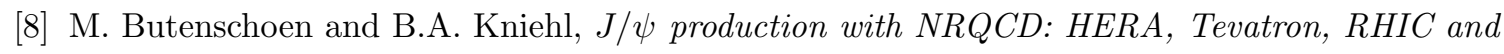
LHC, AIP. Conf. Proc. 1343 (2011) 409 [arXiv:1011.5619] [InSPIRE].

[9] Y.-Q. Ma, K. Wang and K.-T. Chao, Complete next-to-leading order calculation of the $J / \psi$ and $\psi^{\prime}$ production at hadron colliders, Phys. Rev. D 84 (2011) 114001 [arXiv:1012.1030] [InSPIRE].

[10] M. Butenschoen and B.A. Kniehl, World data of $J / \psi$ production consolidate nonrelativistic QCD factorization at next-to-leading order, Phys. Rev. D 84 (2011) 051501 [arXiv: 1105.0820] [InSPIRE].

[11] M. Butenschoen and B.A. Kniehl, Probing Nonrelativistic QCD Factorization in Polarized

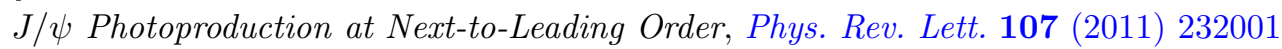
[arXiv:1109.1476] [InSPIRE].

[12] M. Butenschoen and B.A. Kniehl, $J / \psi$ Polarization at the Tevatron and the LHC: Nonrelativistic-QCD Factorization at the Crossroads, Phys. Rev. Lett. 108 (2012) 172002 [arXiv:1201.1872] [InSPIRE].

[13] K.-T. Chao, Y.-Q. Ma, H.-S. Shao, K. Wang and Y.-J. Zhang, $J / \psi$ Polarization at Hadron Colliders in Nonrelativistic QCD, Phys. Rev. Lett. 108 (2012) 242004 [arXiv:1201.2675] [InSPIRE].

[14] M. Butenschoen and B.A. Kniehl, $J / \psi$ production in NRQCD: A global analysis of yield and polarization, Nucl. Phys. Proc. Suppl. 222 (2012) 151 [arXiv:1201.3862] [InSPIRE]. 
[15] B. Gong, L. -P. Wan, J. -X. Wang and H. -F. Zhang, Polarization for Prompt J/ $\psi\left(\psi^{\prime}\right)$ production at the Tevatron and $L H C$, [arXiv: 1205.6682] [InSPIRE]

[16] P. Artoisenet, J.M. Campbell, J.P. Lansberg, F. Maltoni and F. Tramontano, $\Upsilon$ Production at Fermilab Tevatron and LHC Energies, Phys. Rev. Lett. 101 (2008) 152001 [arXiv:0806.3282] [InSPIRE].

[17] Z.-B. Kang, J.-W. Qiu and G. Sterman, Factorization and Quarkonium Production, Nucl. Phys. Proc. Suppl. 214 (2012) 39 [InSPIRE].

[18] Z.-B. Kang, J.-W. Qiu and G. Sterman, Heavy Quarkonium Production and Polarization, Phys. Rev. Lett. 108 (2012) 102002 [arXiv:1109.1520] [InSPIRE].

[19] E. Braaten and T.C. Yuan, Gluon fragmentation into heavy quarkonium, Phys. Rev. Lett. 71 (1993) 1673 [hep-ph/9303205] [InSPIRE].

[20] E. Braaten and T.C. Yuan, Gluon fragmentation into spin-triplet $S$-wave quarkonium, Phys. Rev. D 52 (1995) 6627 [hep-ph/9507398] [InSPIRE].

[21] G.T. Bodwin and J. Lee, Relativistic corrections to gluon fragmentation into spin-triplet S-wave quarkonium, Phys. Rev. D 69 (2004) 054003 [hep-ph/0308016] [InSPIRE].

[22] J.C. Collins and D.E. Soper, Parton Distribution and Decay Functions, Nucl. Phys. B 194 (1982) 445 [InSPIRE].

[23] M. Gremm and A. Kapustin, Annihilation of $S$-wave quarkonia and the measurement of $\alpha_{s}$, Phys. Lett. B 407 (1997) 323 [hep-ph/9701353] [InSPIRE].

[24] L.D. Landau, The moment of a 2-photon system, Dokl. Akad. Nawk., USSR 60 (1948) 207.

[25] C.-N. Yang, Selection Rules for the Dematerialization of a Particle into Two Photons, Phys. Rev. 77 (1950) 242 [InSPIRE].

[26] G.T. Bodwin and A. Petrelli, Order $v^{4}$ corrections to S-wave quarkonium decay, Phys. Rev. D 66 (2002) 094011 [hep-ph/0205210] [InSPIRE].

[27] N. Brambilla, E. Mereghetti and A. Vairo, Hadronic quarkonium decays at order $v^{7}$, Phys. Rev. D 79 (2009) 074002 [Erratum ibid. D 83 (2011) 079904] [arXiv:0810. 2259] [InSPIRE].

[28] G.T. Bodwin, D. Kang and J. Lee, Potential-model calculation of an order- $v^{2}$ nonrelativistic QCD matrix element, Phys. Rev. D 74 (2006) 014014 [hep-ph/0603186] [InSPIRE].

[29] E. Braaten and Y.-Q. Chen, Dimensional regularization in quarkonium calculations, Phys. Rev. D 55 (1997) 2693 [hep-ph/9610401] [InSPIRE].

[30] E. Braaten and J. Lee, Next-to-leading order calculation of the color-octet ${ }^{3} S_{1}$ gluon fragmentation function for heavy quarkonium, Nucl. Phys. B 586 (2000) 427 [hep-ph/0004228] [InSPIRE].

[31] J. Lee, Next-to-leading order calculation of a fragmentation function in a light-cone gauge, Phys. Rev. D 71 (2005) 094007 [hep-ph/0504285] [InSPIRE].

[32] A.C. Hearn, REDUCE User's Manual v. 3.7 (The RAND Corporation, Santa Monica, 1999) [E-mail: reduce@rand.org].

[33] R. Mertig, M. Böhm and A. Denner, Feyn Calc - Computer-algebraic calculation of Feynman amplitudes, Comput. Phys. Commun. 64 (1991) 345 [InSPIRE].

[34] S. Wolfram, Mathematica, third edition (Wolfram Media, Champaign, Cambridge University Press, New York, 1996). 
[35] R. Barbieri, R. Gatto and R. Kogerler, Calculation of the annihilation rate Of $P$ wave quark-antiquark bound states, Phys. Lett. B 60 (1976) 183 [InSPIRE].

[36] R. Barbieri, R. Gatto and E. Remiddi, Singular binding dependence in the hadronic widths of $1^{++}$and $1^{+-}$heavy quark antiquark bound states, Phys. Lett. B 61 (1976) 465 [InSPIRE].

[37] C.-H. Chang, Hadronic production of $J / \psi$ associated with a gluon, Nucl. Phys. B 172 (1980) 425 [InSPIRE].

[38] B. Guberina, J.H. Kühn, R.D. Peccei and R. Rückl, Rare decays of the $Z^{0}$, Nucl. Phys. B 174 (1980) 317 [InSPIRE].

[39] E.L. Berger and D. Jones, Inelastic photoproduction of $J / \psi$ And $\Upsilon$ by gluons, Phys. Rev. D 23 (1981) 1521 [InSPIRE].

[40] A. Petrelli, M. Cacciari, M. Greco, F. Maltoni and M.L. Mangano, NLO production and decay of quarkonium, Nucl. Phys. B 514 (1998) 245 [hep-ph/9707223] [InSPIRE].

[41] A.V. Manohar, Heavy quark effective theory and nonrelativistic QCD Lagrangian to order $\alpha_{s} / m^{3}$, Phys. Rev. D 56 (1997) 230 [hep-ph/9701294] [InSPIRE].

[42] M. Beneke, Non-relativistic effective theory for Quarkonium Production in Hadron Collisions, In "Stanford 1996, The strong interaction, from hadrons to partons" 549-574 [hep-ph/9703429] [InSPIRE].

[43] G.T. Bodwin and Y.-Q. Chen, Renormalon ambiguities in NRQCD operator matrix elements, Phys. Rev. D 60 (1999) 054008 [hep-ph/9807492] [InSPIRE].

[44] Zhi-Guo He, private communication.

[45] P. Cho and A.K. Leibovich, Color-octet quarkonia production, Phys. Rev. D 53 (1996) 150 [hep-ph/9505329] [InSPIRE].

[46] B. Gong, X.Q. Li and J.-X. Wang, QCD corrections to $J / \psi$ production via color octet states at Tevatron and LHC, Phys. Lett. B 673 (2009) 197 [Erratum ibid. B 693 (2010) 612] [arXiv: 0805.4751] [InSPIRE].

[47] G. T. Bodwin, H. S. Chung, D. Kang, J. Lee and C. Yu, Improved determination of color-singlet nonrelativistic QCD matrix elements for S-wave charmonium, Phys. Rev. D 77 (2008) 094017 [arXiv: 0710.0994] [InSPIRE].

[48] G.P. Lepage, A New Algorithm for Adaptive Multidimensional Integration, J. Comput. Phys. 27 (1978) 192 [InSPIRE]. 